\title{
OPTIMAL RESOURCE ALLOCATION FOR VIDEO STREAMING OVER COGNITIVE RADIO NETWORK VIA GEOMETRIC PROGRAMMING
}

\author{
by

\section{BO GUAN} \\ A thesis \\ presented to Ryerson University \\ in partial fulfillment of the \\ requirement for the degree of \\ Master of Applied Science \\ in the Program of \\ Electrical and Computer Engineering
}

Toronto, Ontario, Canada, 2012

(C) Bo Guan, 2012 


\section{Author's Declaration}

I hereby declare that I am the sole author of this thesis. This is a true copy of the thesis, including any required final revisions, as accepted by my examiners.

I authorize Ryerson University to lend this thesis to other institutions or individuals for the purpose of scholarly research.

Author's signature:

I further authorize Ryerson University to reproduce this thesis by photocopying or by other means, in total or in part, at the request of other institutions or individuals for the purpose of scholarly research.

I understand that my thesis may be made electronically available to the public.

Author's signature: 


\section{Borrower's Page}

Ryerson University requires the signatures of all persons using or photocopying this thesis. Please sign below, and give address and date.

\begin{tabular}{|c|c|c|c|}
\hline Name & Signature & Address & Date \\
\hline & & & \\
\hline & & & \\
\hline & & & \\
\hline & & & \\
\hline & & & \\
\hline & & & \\
\hline & & & \\
\hline & & & \\
\hline & & & \\
\hline & & & \\
\hline & & & \\
\hline & & & \\
\hline & & & \\
\hline & & & \\
\hline & & & \\
\hline & & & \\
\hline & & & \\
\hline & & & \\
\hline & & & \\
\hline & & & \\
\hline & & & \\
\hline & & & \\
\hline & & & \\
\hline & & & \\
\hline & & & \\
\hline & & & \\
\hline & & & \\
\hline
\end{tabular}




\title{
ABSTRACT \\ OPTIMAL RESOURCE ALLOCATION FOR VIDEO STREAMING OVER COGNITIVE RADIO NETWORK VIA GEOMETRIC PROGRAMMING
}

\author{
(C) Bo Guan \\ Master of Applied Science, Electrical and Computer Engineering, \\ Ryerson University, Toronto, Canada, 2012
}

Cognitive Radio (CR) is a new paradigm in wireless communications to enhance utilization of limited spectrum resources. In the cognitive radio networks, each secondary user can use wireless channels for data transmission to improve the spectrum utilization. This thesis focus on the resource allocation problem for video streaming over cognitive radio networks, where secondary users and primary users transmit data simultaneously in a common frequency band. Respectively, we investigate CR in both single channel and multiple channels scenarios for single-layered and multi-layered streaming video, which is encoded into multiple layers delivered over a separate channel. Moreover, the source rate, the transmission rate, and the transmission power at each video session in each channel are jointly optimized to provide Quality of Service (QoS) guarantee to all video sessions in the secondary network. The optimization problem is formulated into a Geometric Programming (GP) problem, which can be solved efficiently. In the simulations, we demonstrate that the proposed scheme can achieve a lower Packet Loss Rate (PLR) and queuing delay, thus leading to a higher video quality for the video streaming sessions, compared to the uniform scheme. 


\section{Acknowledgments}

First and foremost I am deeply indebted to my advisor Dr. Yifeng He for his continuous guidance and encouragement throughout my research. I am very grateful to Dr. He for his insightful suggestions, valuable discussions, and great attention to details. This work would have been impossible without their feedback, patience and kindness.

I would like to thank the Electrical and Computer engineering Department of Ryerson University for providing well-equipped facilities and professional support by Ryerson Communication Laboratory (RCL) and Ryerson Multimedia Laboratory (RML). I am very thankful to my thesis committee members for invaluable advice on the thesis.

I express my appreciation to the members of Ryerson Multimedia Research Laboratory and Ryerson Communication Laboratory for their help and cooperation. It is enjoyable and a great pleasure to work in such a friendly and collaborative environment 


\section{Table of Contents}

Abstract

1. Introduction 1

1.1 Background Study 1

1.1.1 Cognitive Radio 1

1.1.2 Wireless Video Streaming 3

1.2 Problems and Objectives 5

1.3 Technical Challenges and Related Work 7

1.4 Thesis Contributions 10

$\begin{array}{lll}1.5 & \text { Thesis Organization } & 10\end{array}$

2. Background 12

2.1 Cognitive Radio Networks 12

2.2 Spectrum Underlay versus Spectrum Overlay 15

2.2.1 Spectrum Underlay $C R \quad 15$

2.2.2 Spectrum Overlay CR 17

2.3 Video streaming over Cognitive Radio 20

2.4 Geometric Programming 24

2.4.1 Monomial and Posynomial Functions 25

2.4.2 Standard Form Geometric Program 26

3. Single Layer Video Streaming over Spectrum Underlay CR Networks 28

3.1 Problem Statement 28

3.2 System Models 29

3.2.1 Network Topology 29

3.2.2 Queuing Model 30

3.2.3 CMDA Model 32

3.2.4 QoS Metrics 33

3.3 Optimal Resource Allocation 34

3.3.1 Problem Formulation 34

3.3.2 Optimal Solution 38

3.4 Simulations $\quad 40$

3.4.1 Simulation Setting $\quad 40$

3.4.2 Simulation Results 41

3.5 Chapter Summary 45

4. Multi-Layered Video Streaming over Multi-Channel CR Networks 47 
4.1 Problem Statement 47

4.2 Multi-Channel CR System Models 48

4.2.1 Network Topology 48

4.2.2 Rate Distortion Model for Layered Video 50

4.2.3 Queuing Model 51

4.2.4 Transmission Model 52

4.3 Optimal Resource Allocation 53

4.3.1 Problem Formulation 53

4.3.2 Optimal Solution 56

4.4 Simulations $\quad 50$

4.4.1 Simulation Setting 57

4.4.2 Simulation Results 58

4.5 Chapter Summary $\quad 61$

5. Conclusion and Future Work 62

5.1 Conclusion $\quad 62$

5.2 Future Works 63

$\begin{array}{ll}\text { Bibliography } & 64\end{array}$

$\begin{array}{ll}\text { A List of Publications } & 70\end{array}$ 


\section{List of Figures}

3.1 Illustration of a spectrum underlay cognitive radio network 30

3.2 Block diagram of the sender of a secondary session 31

3.3 Comparison of PLR between the proposed scheme and uniform scheme 42

3.4 Comparison of queuing delay between the proposed scheme and the uniform scheme 42

3.5 Comparison of frame PSNR of Forman CIF sequence between the proposed scheme and the uniform scheme 44

3.6 Variation of video source rates with the quality weight of secondary session $1 \quad 49$

4.1 Illustration of multi-layered video streaming over multi-channel cognitive radio networks 42

4.2 Comparison of PLR between the optimal scheme and the equal scheme 59

4.3 Comparison of frame PSNR of Forman CIF sequence between the optimal scheme $\begin{array}{ll}\text { and equal scheme } & 60\end{array}$ 


\section{List of Tables}

4.1 Comparison between actual distortion and estimated distortion for FORMAN CIF sequence 


\section{Chapter 1}

\section{Introduction}

\subsection{Background Study}

\subsubsection{Cognitive Radio}

THE need to wirelessly share high-quality multimedia content is driving the need for ever-increasing wireless transport capacity, which is, however, limited by the scarcity of the available radio spectrum. The current wireless networks follow a static spectrum assignment strategy where radio spectrum are allocated to license holders for exclusive usage privileges on a long-term basis for large geographical regions. With the increase in spectrum demand, this strategy has been challenged by the problem of spectrum scarcity, which makes the radio spectrum one of the most heavily regulated and expensive natural resources around the world. Achieving high spectrum utilization is, therefore, one of the most critical research objectives in designing wireless communication systems today. In fact, as discussed in a report by Federal Communication Commission (FCC) on spectrum usage, the spectrum utilization varies from $15 \%$ to $85 \%$, depending on the geographical 
area [1].The limited available spectrum and inefficient spectrum utilization necessitate a new mechanism to exploit the existing spectrum in an opportunistic manner. Consequently, Cognitive Radio (CR) is proposed to solve this inefficiency problem in spectrum usage $[2,3]$.

Cognitive radio is a new paradigm in wireless communication which can change its transmission parameters based on what it learns from the environment in which it operates [4]. It is defined as a radio that is able to utilize available side information, in a decentralized paradigm, in order to efficiently use the radio spectrum left unused by licensed systems. Opportunistic users (referred to secondary users in this thesis) may dynamically select best available channels, and adapt their transmission parameters to avoid harmful interference between contending users. Furthermore, Medium Access Control (MAC) protocols exploit sensing stimuli to build up a spectrum opportunity map (cognitive sensing). It has an important role in four major cognitive radio functions: spectrum mobility, channel sensing, resource allocation, and spectrum sharing [2]. CR techniques exploit spectrum opportunities in space, time, and frequency while protecting users of the primary networks from excessive interference due to spectrum access from the users of secondary networks [5]. 
The spectrum utilization techniques in cognitive radio networks can be classified into two classes: spectrum overlay and spectrum underlay $[6,7]$. In the spectrum overlay scheme, secondary users are only allowed to access the spectrum channels of the primary network that are not being used by primary users. In the spectrum underlay scheme, primary users and secondary users can transmit data simultaneously over the same channel as long as the aggregated interference to the primary user generated by secondary users are below an acceptable threshold [8]. Compared to spectrum overlay, the advantage of spectrum underlay is that the secondary users can directly access the licensed spectrum without considering the behaviors of the primary users. In the spectrum underlay scheme, the transmission power and transmission rate of each secondary user become critically important in order to guarantee the interference to the primary network below an acceptable threshold.

\subsubsection{Wireless Video Streaming}

The past few years have seen a proliferation use of various multimedia applications over wired packet networks, such as interactive voice communications (Voice over Internet Protocol (VoIP)), multimedia messaging, video conferencing, live TV broadcasting and on-demand multimedia streaming, e.g., Youtube, Hulu, NetFlix. In the 
meantime, the rapid diffusion of wireless devices in the past years, such as iPad, iPod, iPhone, is increasingly propelling the shift of users toward wireless technologies.

From a communication point of view, wireless video streaming can be further divided into two classes:

1. $\quad$ Live video streaming, such as TV broadcasting;

2. On-demand streaming, also called video-on-demand (VoD), such as remote education, Youtube.

The major difference between the two groups is that the latter requires some form of interactions between the source and end hosts [9]. In specific, in the first group, the video packets are delivered one way from the source to destinations. All the users subscribed to the service receive the same media content and process the playback at the same time. The latter group, however, allows users to select different media contents from a rich media database and interactively control the playback. With more flexibility rendered to users, of the many applications of distributed media streaming systems, VoD has much appeal.

With different characteristics of media service, the QoS provision schemes for the two different media applications are also diverse. The live streaming service requires minimum start-up delay to ensure the instant information delivery from the source. In this 
case, the media buffer is kept small to reduce the latency of start-up; therefore, the media traffic requires static throughput support with persistent guaranteed data rate. The ondemand media streaming, however, can tolerate a fairly long start-up delay compared with the live streaming service, as long as the media can be smoothly played without interruptions. In other words, the buffer plays an important role now and how to make use of the playback buffer in network resource allocation becomes critical. With abundant buffer storage, the users are resilient to the network dynamics which could benefit the resource allocation. Moreover, it is important to note that the video-ondemand allows users to arbitrarily select the position of media playback. Once the user switches to a new playback position, its previous buffer contents become useless and new content must be pulled immediately via the network. This may require intensive network resource in a short period.

\subsection{Problems and Objectives}

Driven by the ever-growing demand of wireless multimedia services and increasing importance of CR networks in wireless communication, in this thesis, we will focus on the problem of resource allocation, which is employed to provide reliable video streaming service to secondary users according to QoS requests. We first explore one 
basic single channel CR network model based on Code Division Multiple Access (CDMA) technology, and investigate QoS provisioning for secondary video streaming session. In addition, we expanded it into a more sophisticated scenario - multi-layered video streaming over multi-channel $\mathrm{CR}$ network, and demonstrated that the issued methodology could provide convincing multimedia streaming quality. The proposed methods can expand the potential value-added service for ISPs, such as video conferencing, TV broadcasting and wireless on-demand multimedia streaming $[5,10,11]$.

Moreover, because of the unique feature of $\mathrm{CR}$ networks and specific QoS requirements of video streaming services, existing scheme designed for video streaming over traditional networks could not be easily extended, and we indicate our specific key research objectives of this work as follows.

1. QoS Provisioning for Secondary Users. The QoS supports depend on the resource allocation at each user and in each channel, which is a nontrivial task. The secondary users need to carefully select their transmission power and transmission rate to guarantee that they do not generate an unacceptable interference to the primary users. In this work, we present the QoS metrics, the Packet Loss Rate (PLR) for the congestion PLR due to the queue overflow, the transmission errors, and Queuing 
Delay (QD), which is defined as the duration from the time when packet arrives in the queuing system to the time when the packet leaves the queuing system.

2. Interferences among Secondary Users. The interferences among the users may corrupt the video packets, thus degrading the video quality. Existing methods for solving this interference problem leave uncertainties and difficulties in practice for the cognitive radio applications. We try to solve such interference problems in spectrum underlay cognitive radio networks.

3. Resource Allocation for Secondary User. The resource allocation to the secondary user needs to be controlled appropriately to guarantee undisturbed operation of primary users. We formulate this resource allocation problem into a Geometric Programming (GP) problem, which can be solved efficiently.

\subsection{Technical Challenges and Related Work}

Although wireless networks provide a low-cost and flexible infrastructure to multimedia applications, this infrastructure is unreliable and provides dynamically varying resources with only limited QoS support. Moreover, media streaming applications, such as TV broadcasting, have especial stringent QoS requirements compared with genetic data communications [13]. In particular for cognitive radio 
networks, media traffic is characterized by strong time sensitivity and inelastic bandwidth requirement. It is challenging to provide QoS guarantee for video streaming over designated spectrum underlay cognitive radio networks.

The emerging high-speed wireless access technologies and the requirements of different wireless applications are expected to create a huge demand on spectral resources in the next generation wireless communication systems. Due to the rapidly increasing demand of multimedia application over wireless networks, video streaming services have become an urgent problem attracting significant efforts among various research communities, from networking society to signal processing society. Meanwhile, CDMA has been adopted as multiple access technology for $3 \mathrm{G}$ and beyond due to its advantages such as universal frequency reuse, soft handoff, inherent diversity, and high spectrum efficiency [12]. Multimedia services over IP-based CDMA wireless networks will be one of the key applications in cognitive radio field.

Resource allocation in single-channel spectrum underlay cognitive radio networks was investigated in [14]. Video streaming over cognitive radio networks was investigated in [16-17]. In [16], Hu et al. adopted scalable videos to accommodate heterogeneous channel availabilities and dynamic network conditions in spectrum overlay cognitive radio networks. In [17], Li et al. optimally allocated the channels to maximize the overall 
network throughput while providing users with the smooth video playback. In this thesis, we optimized the resource allocation to provide QoS guarantee for video streaming over single-channel cognitive radio networks. Furthermore, we introduced the detail methodology in the following chapters, and also bring along with the multi-layer video streaming over multi-channel cognitive radio, for more practical network scenarios.

Many milestones, both regulation and technical, have been reached in opening spectrum for more flexible and efficient use, and this trend will continue. CR technology plays a significant role in making the best use of scarce spectrum to support fast growing demand for wireless applications, ranging among Wireless IPTV (in which TV programming is transmitted to smart terminals), Vehicle Asset Tracking and Control, Smart Grid for utilities, transportation and private companies, which are all applications or consumer of these services [9]. Starting with the lowest hanging fruit, wireless providers now see a market in niche areas that need more flexibility in radio spectrum. For example, TerraStar [9] is a satellite communications company that has recently launched a mobile product that combines satellite and cellular communication onto one smart phone, built with Software Define Radios (SDR) that will enable both types of communications. Potential market for these versatile phones is outdoor enthusiasts, local emergency first responders, and transportation companies [9]. As mentioned above the 
opportunities for agile radio technologies like cognitive radio systems are directly linked to the growing market for wireless data transmission as $3 \mathrm{G}$ applications grow to $4 \mathrm{G}$.

\subsection{Thesis Contributions}

Our work consists of the resource allocation problem for video streaming over cognitive radio networks. Motivated by the aforementioned challenges in this field, the proposed work aims to provide QoS guarantee for video streaming over multi-channel spectrum underlay cognitive radio networks by optimizing the resource allocation using geometric programming method. We jointly optimize the source rate, the transmission rate, and the transmission power at each video session to provide QoS guarantee to all video sessions in the secondary network. It is expected to benefit the advancement of next generation wireless communication technologies and the applications in communication and information technology. The presented methods can be applied into many applications, such as VOD, video conferencing, IPTV.

\subsection{Thesis Organization}

This thesis is proposed of five chapters and the rest of this thesis is organized as follows: 
Chapter 2: Background, reviews the background and related works reported in the literatures for cognitive radio network and video streaming.

Chapter 3: Single Layer Video Streaming over Spectrum Underlay CR Networks, conducts a QoS optimization method using Geometric Programming for video streaming over spectrum underlay cognitive radio. Chapter 3 presents the work for the single layer video streaming over spectrum underlay CR networks.

Chapter 4: Multi-Layered Video Streaming over Multi-Channel CR Networks, discusses a more sophisticated scenario, based on the model in Chapter 3. The multi-layered video streaming over multi-channel cognitive radio network is studied to find a more efficient way of utilizing resource for secondary users with multi-channel cognitive radio.

Chapter 5: Conclusions and Future Work, discusses the results from our experiment and summarizes the contributions of this work. Future works are also presented. 


\section{Chapter 2}

\section{Background}

WITH the emerging high-speed wireless access technologies, the requirements of different wireless applications are expected to create a huge demand on spectral resources in the next generation wireless systems. Achieving high spectrum utilization is, therefore, one of the most critical research objectives in designing wireless communication systems today. Therefore, there is an increasing interest in developing efficient methods for spectrum management and sharing. Cognitive Radio (CR) is a new paradigm in wireless communications to enhance utilization of limited spectrum resources. The main idea contained in CR technology is that the SU can exploit temporarily and local available licensed spectrum and adapt its radio parameter to opportunistically communicate over the spectrum. As to the detail of the methodologies for cognitive radio, it will be introduced in this chapter.

\subsection{Cognitive Radio Networks}


Cognitive radio technology is a key technology that enables next generation network to use spectrum in a dynamic manner. The CR, which was first proposed by Mitola in 1999 [20], is a promising approach to achieve dynamic spectrum sharing [5]. The term, cognitive radio, can formally be defined as follows [4]: "A Cognitive Radio is a radio that can change its transmitter parameters based on interaction with the environment in which it operates." From this definition, two main characteristics of the cognitive radio can be defined [5, 21]: first conception, Cognitive capability refers to the ability of the radio technology to capture or sense the information from its radio environment. This capability cannot simply be realized by monitoring the power in some frequency band of interest but more sophisticated techniques are required in order to capture the temporal and spatial variations in the radio environment and avoid interference to other users. Through this capability, the portions of the spectrum that are unused at a specific time or location can be identified. Consequently, the best spectrum and appropriate operating parameters can be selected. Second conception, Reconfigurability enables the radio to be dynamically programmed according to the radio environment. More specifically, the cognitive radio can be programmed to transmit and receive on a variety of frequencies and to use different transmission access technologies supported by its hardware design. 
The objective of the cognitive radio is to exploit the best available spectrum through cognitive capability and reconfigurability as described before. Since most of the spectrum is already assigned, the most important challenge is to share the licensed spectrum without interfering with the transmission of other licensed users. The cognitive radio enables the usage of temporally unused spectrum, which is referred to as white space [4]. If a licensed user further uses this band, the cognitive radio moves to another spectrum hole or stays in the same band, altering its transmission power level or modulation scheme to avoid interference. In [22], opportunistic networks are divided, according to the type of infrastructure, in centralized and distributed networks. Centralized networks are then classified depending on whether the Access Point controller takes part in data transmission among the secondary users. If the controller doesn't take over the SUs' transmission, it will be classified as the decentralized scheme. Then decentralized networks are classified according to how signaling and channel negotiation are managed by the network. It is defined as a radio that is able to utilize available side information, in a decentralized paradigm, in order to efficiently use the radio spectrum left unused by licensed systems. CR techniques exploit spectrum opportunities in space, time, frequency, while protecting users of the primary network from excessive interference due to spectrum access from the users of secondary networks. 
In this first place, the CR techniques deal with the underlay and overlay operation of cognitive radios. On the one hand, the underlay paradigm mandates that concurrent primary and secondary transmissions may occur as long as the aggregated interference generated by the SUs is below some acceptable threshold [20]. On the other hand, the overlay paradigm allows the coexistence of simultaneous primary and secondary communications in the same frequency channel, as long as the SUs somehow facilitate transmission of the PUs, for example by means of advanced coding or cooperative techniques. In particular, in a cooperative scenario the SUs could decide to assign part of their power to their own secondary communications and the remaining power to relay the PUs' transmission [5]. Recent research focus on the hybrid CR system where underlay and overlay CR approaches are combined. Several methods proposed on occasional switches from an overlay $\mathrm{CR}$ mode to an underlay $\mathrm{CR}$ mode enable to maximize the average throughput of a secondary network and stable transmission of a secondary user.

\subsection{Spectrum Underlay versus Spectrum Overlay}

\subsubsection{Spectrum Underlay $C R$}

In spectrum underlay secondary users are allowed to transmit their data in the licensed spectrum band when primary users are also transmitting. The interference temperature 
model is imposed on secondary users' transmission power so that the interference at a primary user's receiver is within the interference temperature limit and primary users can deliver their packet to the receiver successfully. Spread spectrum techniques are usually adopted by secondary users to fully utilize the wide range of spectrum. However, due to the constraints on transmission power, secondary users can only achieve short-range communication. If primary users transmit data all the time in a constant mode, spectrum underlay does not require secondary users to perform spectrum detection to find available spectrum band.

In the spectrum overlay paradigm, secondary users are only allowed to access spectrum resources (i.e., channels) owned by the primary network provider if these channels are not being used by primary users. Here, spectrum sensing needs to be performed to avoid possible collision with primary users [1], [12]. Given that the spectrum opportunities are detected by a spectrum sensing technique, it is important to coordinate the spectrum sharing among secondary users in such a way that different design objectives can be achieved. Centralized architectures for spectrum sensing and sharing in cognitive wireless networks were proposed in [6] and [7]. In general, a medium access control (MAC) layer protocol with sensing capability is important to 
perform fair resource allocation among secondary users while avoiding collision with primary users.

In an underlay system, regulated spectral masks impose stringent limits on radiated power as a function of frequency, and perhaps location. Radios coexist in the same band with primary licensees, but are regulated to cause interference below prescribed limits. For example, a low-powered radio could coexist in the same frequency channel with a high-powered broadcast radio. Because of the power limitation, underlay radios (UR) must spread their signals across large bandwidths, and/or operate at relatively low rates. The underlay approach imposes severe constraints on the transmission power of secondary users so that they operate below the noise floor of primary users. By spreading transmitted signals over a wide frequency band (UWB), secondary users can potentially achieve short-range high data rate with extremely low transmission power. Based on a worst-case assumption that primary users transmit all the time, this approach does not rely on detection and exploitation of spectrum white space.

\subsubsection{Spectrum Overlay CR}

Spectrum overlay is also referred to as opportunistic spectrum access. Unlike spectrum underlay, secondary users in spectrum overlay will only use the licensed spectrum when primary users are not transmitting, so there is no interference temperature limit imposed 
on secondary users' transmission. Instead, secondary users need to sense the licensed frequency band and detect the spectrum white space, in order to avoid harmful interference to primary users.

For the spectrum underlay paradigm, it is required that an interference limit corresponding to an interference temperature level be maintained at receiving points of the primary network. A graph-theoretic model for spectrum sharing/access among secondary users was proposed in [8] where different objective functions were investigated. In [9], the channel allocation problem was formulated using game theory. Here, the proposed utility functions capture the interference perceived by one user on each channel and/or the interference this user creates to its neighboring users. In these papers, primary users were not explicitly protected from interference due to spectrum access by secondary users. In [13], a heuristic based channel and power allocation algorithm was proposed where interference constraints for primary users were considered. However, how good the performance of the proposed algorithm compared to the optimal solution was not known. In [14], a user removal algorithm based on the treepruning algorithm was proposed so that QoS constraints for secondary users and interference temperature constraints for primary users are satisfied. The proposed removal algorithm is, however, computationally extensive. 
In the spectrum overlay paradigm, secondary users are only allowed to access spectrum resources (i.e., channels) owned by the primary network provider if these channels are not being used by primary users. Here, spectrum sensing needs to be performed to avoid possible collision with primary users [27, 28]. Given that the spectrum opportunities are detected by a spectrum sensing technique, it is important to coordinate the spectrum sharing among secondary users in such a way that different design objectives can be achieved. Centralized architectures for spectrum sensing and sharing in cognitive wireless networks were proposed in [29, 30]. In general, a MAC layer protocol with sensing capability is important to perform fair resource allocation among secondary users while avoiding collision with primary users.

Spectrum overlay can be applied in either temporal or spatial domain [31]. In the former, secondary users aim to exploit temporal spectrum opportunities resulting from the burst traffic of primary users. In the latter, secondary users aim to exploit frequency bands that are not used by primary users in a particular geographic area. A typical application is the reuse of certain TV-bands that are not used for TV broadcast in a particular region. In the TV broadcast system, TV-bands assigned to adjacent regions are different to avoid co-site interference. This results in unused frequency bands varying over space. In general, spectrum opportunities vary in both temporal and spatial domains. 
It is often assumed in the literature that one variation is at a much slower scale than the

other.

Initial attempts at addressing the identification and exploitation of temporal spectrum opportunities that also vary in space can be found in [37]. For an extended overview of challenges and recent developments in spectrum overlay, readers are referred to [34].

\subsection{Video Streaming over Cognitive Radio}

Streaming video is multimedia that is constantly received by and presented to an enduser while being delivered by a streaming provider [48]. The name refers to the delivery method of the medium rather than to the medium itself. The distinction is usually applied to media that are distributed over telecommunications networks, as most other delivery systems are either inherently streaming (e.g., radio, television) or inherently nonstreaming (e.g., books, video cassettes, audio CDs). Internet television is a commonly streamed medium. Streaming media can be something else other than video and audio. Live closed captioning and stock tickers are considered streaming text, as is Real-Time Text. Live streaming, delivering live over the Internet, involves a camera for the media, an encoder to digitize the content, a media publisher, and a content delivery network to distribute and deliver the content. 
Designing a network protocol to support streaming video raises many issues, such as:

1. Datagram protocols, such as the User Datagram Protocol (UDP), send the video stream as a series of small packets. This is simple and efficient; however, there is no mechanism within the protocol to guarantee delivery. It is up to the receiving application to detect loss or corruption and recover data using error correction techniques. If data is lost, the stream may suffer a dropout.

2. The Real-time Streaming Protocol (RTSP), Real-time Transport Protocol (RTP) and the Real-time Transport Control Protocol (RTCP) were specifically designed to stream media over networks. RTSP runs over a variety of transport protocols, while the latter two are built on top of UDP. HTTP adaptive bitrate streaming, which is based on HTTP progressive download, but contrary to the previous approach, here the files are very small, so that they can be compared to the streaming of packets, much like the case of using RTSP and RTP [49].

3. Reliable protocols, such as the Transmission Control Protocol (TCP), guarantee correct delivery of each bit in the media stream. However, they accomplish this with a system of timeouts and retries, which makes them more complex to implement. It also means that when there is data loss on the network, the media stream stalls while the protocol handlers detect the loss and retransmit the missing data. Clients can minimize 
this effect by buffering data for display. While delay due to buffering is acceptable in video on demand scenarios, users of interactive applications such as video conferencing will experience a loss of fidelity if the delay that buffering contributes to exceeds $200 \mathrm{~ms}$ [50].

4. Unicast protocols send a separate copy of the media stream from the server to each recipient. Unicast is the norm for most Internet connections, but does not scale well when many users want to view the same television program concurrently.

5. Multicast protocols were developed to reduce the data replication (and consequent server/network loads) that occurs when many recipients receive unicast content streams independently. These protocols send a single stream from the source to a group of recipients. Depending on the network infrastructure and type, multicast transmission may or may not be feasible. One potential disadvantage of multicasting is the loss of video on demand functionality. Continuous streaming of radio or television material usually precludes the recipient's ability to control playback. However, this problem can be mitigated by elements such as caching servers, digital set-top boxes, and buffered media players. IP Multicast provides a means to send a single media stream to a group of recipients on a computer network. A multicast protocol, usually Internet Group 
Management Protocol, is used to manage delivery of multicast streams to the groups of recipients on a LAN.

6. Peer-to-peer $(P 2 P)$ protocols arrange for prerecorded streams to be sent between computers. This prevents the server and its network connections from becoming a bottleneck. However, it raises technical, performance, quality, and business issues.

Compared with the data transfer applications, video streaming like VoD is characterized by the strong time sensitivity and inelastic bandwidth requirements. The video packets must be downloaded and available at the video decoder before their playback time to allow a continuous video reconstruction. Nevertheless, the CR networks are typically highly dynamic which poses significant challenges to the smooth video streaming. Specifically, in CR networks, primary users (PUs) normally behave in a purely random and unpredictable fashion, making the channel spectrum opportunities for secondary users (SUs) highly variable. Coupled with the dynamic channel status and internal transmission contentions among themselves, the download throughput of SUs is extraordinarily turbulent and intensely changing all the time, which severely affects the quality of the video play-out.

Video streaming over cognitive radio networks was investigated in $[5,10,11]$. There is extensive literature striving to provision guaranteed QoS [38] to users in the dynamic CR 
networks. In [39], it describes a live video streaming system over the infrastructure-based CR networks, in which multiple video flows are delivered to different groups of users. To provide high-quality video streaming with a low video distortion, authors propose a cross-layer optimization scheme which jointly considers coding rate, channel selection and channel sharing. In [5], $\mathrm{Hu}$ et al. adopted scalable videos to accommodate heterogeneous channel availabilities and dynamic network conditions in spectrum overlay cognitive radio networks. In [6], Li et al. optimally allocated the channels to maximize the overall network throughput while providing users with the smooth video playback. In our previous work [7], we optimized the resource allocation to provide QoS guarantee for video streaming over single-channel cognitive radio networks.

\subsection{Geometric Programming}

A geometric program (GP) is a type of mathematical optimization problem characterized by objective and constraint functions that have a special form [40]. The importance of GPs comes from two relatively recent developments: first, new solution methods can solve even large-scale GPs extremely efficiently and reliably; second, a number of practical problems, particularly in electrical circuit design, have recently been found to be equivalent to (or well approximated by) GPs. Putting these two together, we 
get effective solutions for the practical problems. GP is a class of nonlinear optimization with many useful theoretical and computational properties. GP substantially broadens the scope of Linear Programming (LP) applications, and is naturally suited to model several types of important nonlinear systems in science and engineering.

\subsubsection{Monomial and Posynomial Functions}

There are two equivalent forms of GP: standard form and convex form. The first is a constrained optimization of a type of function called posynomial, and the second form is obtained from the first through a logarithmic change of variable. Standard form GP is often used in network resource allocation problems, and convex form GP in problems based on stochastic models such as information theoretic problems. Next before defining the two forms, we first introduce the concepts of monomial and posynomial.

Definition 1 (monomial): A function $\mathrm{f}: \mathrm{R}^{\mathrm{n}} \rightarrow \mathrm{R}$ is a monomial if it has the following expression [40]

$$
\mathrm{g}(\mathrm{x})=c \mathrm{x}_{1}^{\mathrm{a}^{(1)}} \mathrm{x}_{2}^{\mathrm{a}^{(2)}} \cdots \mathrm{x}_{\mathrm{n}}^{\mathrm{a}^{(\mathrm{n})}}
$$

where the multiplicative constants $c \geq 0$ and the exponential constants $a^{(j)} \in R, j=$ $1,2, \ldots \mathrm{n}$.

Definition 2 (posynomial): A function $\mathrm{f}: \mathrm{R}^{\mathrm{n}} \rightarrow \mathrm{R}$ is a posynomial if it is a sum of monomial, as the following below [40] 


$$
f(x)=\sum_{m=1}^{M} c_{m} x_{1}^{a_{m}^{(1)}} x_{2}^{a_{m}^{(2)}} \ldots x_{n}^{a_{m}^{(n)}}
$$

Where $c_{m}, m=1,2, \ldots M$ are nonnegative and $a_{m}^{(j)} \in R, j=1,2, \ldots n, m=1,2, \ldots M$.

\subsubsection{Standard Form Geometric Program}

Standard GP is the optimization problem with posynomial objective function and constraints including posynomial inequalities with upper bound and monomial equalities. A geometric program is an optimization problem of the form

$$
\begin{aligned}
& \text { Minimize } \mathrm{f}_{0}(\mathrm{x}) \\
& \text { Subject to } \mathrm{f}_{\mathrm{i}}(\mathrm{x}) \leq 1 \quad \forall \mathrm{i} \\
& \mathrm{g}_{\mathrm{l}}(\mathrm{x})=1 \quad \forall \mathrm{l}
\end{aligned}
$$

where $g_{l}(x), f_{i}(x)$ are monomial and posynomial defined in (1) and (2), respectively: $f_{i}(x)$ are posynomial functions, $\mathrm{g}_{\mathrm{l}}(\mathrm{x})$ are monomials, and $\mathrm{x}$ are the optimization variables. (There is an implicit constraint that the variables are positive, i.e., $\mathrm{x}>0$.) We refer to the problem (3) as a geometric program in standard form. In a standard form GP, the objective must be posynomial (and it must be minimized); the equality constraints can only have the form of a monomial equal to one, and the inequality constraints can only have the form of a posynomial less than or equal to one. We can switch the sign of any of the exponents in any monomial term in the objective or constraint functions, and still 
have a GP. But if we change the sign of any of the coefficients, or change any of the additions to subtractions, the resulting problem is not a GP. 


\section{Chapter 3}

\section{Single Layer Video Streaming over Spectrum Underlay CR Networks}

IN this chapter, we investigate the resource allocation problem for single layer video streaming over spectrum underlay cognitive wireless networks. Specifically, we jointly optimize the source rate, the transmission rate, and the transmission power at each secondary video session to provide Quality of Service (QoS) guarantee to the delivery of video streams. The optimization problem is formulated into a Geometric Programming (GP), which can be solved efficiently.

\subsection{Problem Statement}

We investigate the resource allocation problem for video streaming over spectrum underlay cognitive radio networks where secondary users and primary users transmit data simultaneously in a common frequency band. It is challenging to obtain a high quality for video streaming over spectrum underlay cognitive radio networks. First, the secondary users need to carefully select their transmission power and transmission rate to guarantee 
that they do not generate an unacceptable interference to the primary users. Second, the secondary users suffer from the interferences from both the primary users and the other secondary users, which may corrupt the video packets. We formulate the resource allocation problem into an optimization problem, which jointly optimizes the source rate, the transmission rate, and the transmission power at each secondary session to provide QoS guarantee to the video streaming sessions. This problem is then turned into an optimal GP problem.

\subsection{System Models}

\subsubsection{Network Topology}

We consider the hierarchical spectrum sharing problem in a spectrum underlay cognitive wireless network where several secondary users and primary users transmit data in a common frequency band (e.g., as in a CDMA-based wireless network) [41]. A communication session is established between a pair of users who wish to communicate with each other. The communication sessions between the primary users are referred to as primary sessions, and the communication sessions between the secondary users are referred to as secondary sessions. The transmission setting considered in this thesis is illustrated in Figure 3.1. The set of primary sessions is denoted by M, and the set of the 
secondary sessions is denoted by N. In this work, we assume that all the secondary sessions in the set $\mathrm{N}$ are video streaming sessions and they are all single-hop sessions.

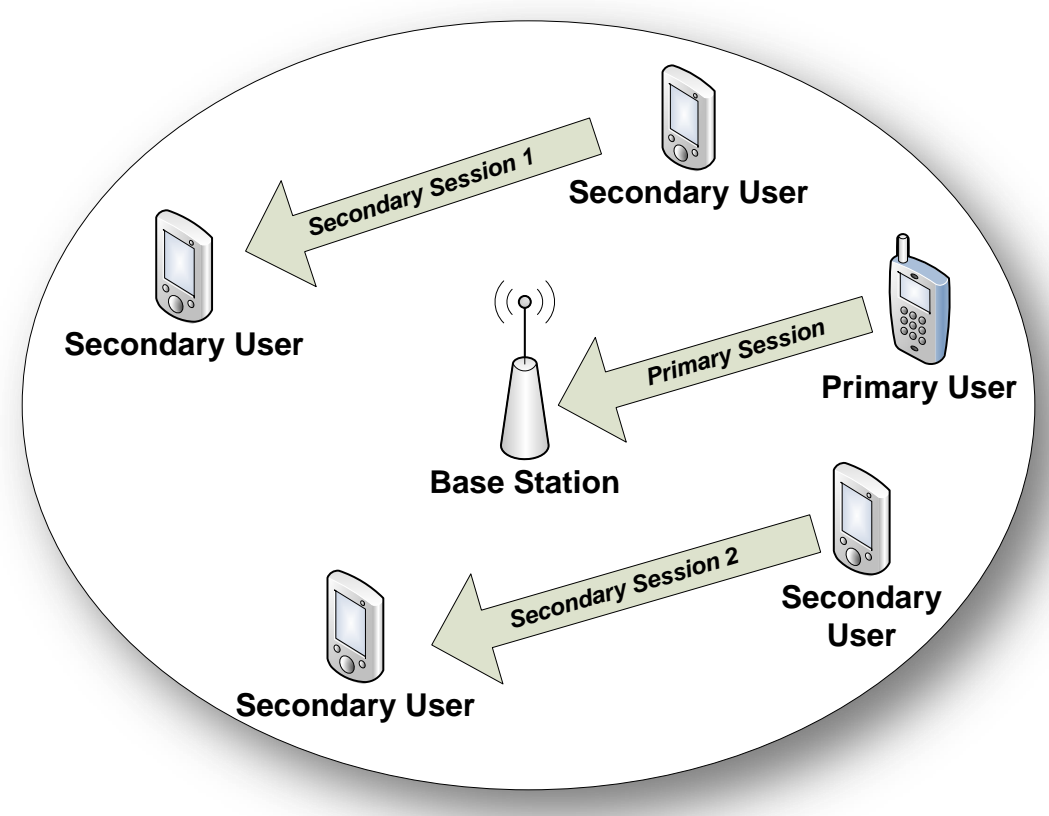

Figure 3.1 Illustration of a spectrum underlay cognitive radio network

Simultaneous communications of primary sessions and secondary sessions will interfere with each other. We will optimize the resource allocation to guarantee that: 1) the interference received at the receiving nodes of the primary sessions should be below an acceptable level, and 2) the secondary sessions (e.g., video streaming sessions) should meet the QoS requirements.

\subsubsection{Queuing Model}

Each secondary session consists of a sender and a receiver. The sender captures and encodes the live video, and then transmits the compressed stream to the receiver. The 
block diagram of the sender of the secondary session $m(\forall m \in M)$ is shown in Figure 3.2 An M/M/1 queuing system [42] is used to buffer the compressed video streams before they are transmitted to the receiver via the wireless channel. The compressed video packets enter the $\mathrm{M} / \mathrm{M} / 1$ queue at a source rate $\mathrm{S}_{\mathrm{m}}$. The video packets are scheduled based on First-In-First-Out (FIFO) order, and are transmitted to the receiver at the transmission rate $R_{m}$ with the transmission power $P_{m}$

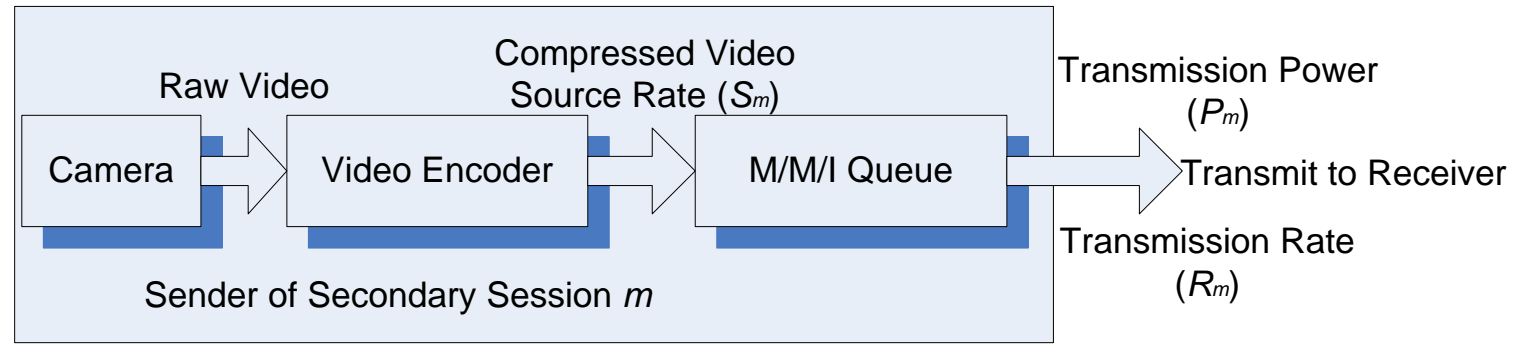

Figure 3.2 Block diagram of the sender of a secondary session

Let $\mathrm{L}_{\mathrm{m}}$ denote the average packet length at the sender of the secondary session $\mathrm{m}$. The packet arrival rate $\tau_{\mathrm{m}}$ to the $\mathrm{M} / \mathrm{M} / 1$ queue is given by $\tau_{\mathrm{m}}=\mathrm{S}_{\mathrm{m}} / \mathrm{L}_{\mathrm{m}}$, and the packet departure rate out of the $M / M / 1$ queue is given by $\mu_{m}=R_{m} / L_{m}$. The $M / M / 1$ queue needs to satisfy the follow condition in order to be stable [42]:

$$
\tau_{\mathrm{m}} \leq \mu_{\mathrm{m}}, \quad \forall \mathrm{m} \in \mathrm{M},
$$

where $\mathrm{M}$ is the set of the secondary sessions.

In the $\mathrm{M} / \mathrm{M} / 1$ queuing system, the tail probability is defined as the probability that the number of packets in the system is larger than a threshold $\varepsilon_{\mathrm{m}}$ [42]. Let the threshold $\varepsilon_{\mathrm{m}}$ 
represent the length of the queue at the secondary session $\mathrm{m}$. The tail probability represents the packet drop probability due to queue overflow. The tail probability $\mathrm{P}_{\mathrm{m}}^{\mathrm{c}}$ at the secondary session $\mathrm{m}$ is given by [42]

$$
\mathrm{P}_{\mathrm{m}}^{\mathrm{c}}=\mathrm{P}_{\mathrm{r}}\left(\mathrm{N}_{\mathrm{m}}>\varepsilon_{\mathrm{m}}\right)=\left(\frac{\tau_{\mathrm{m}}}{\mu_{\mathrm{m}}}\right)^{\left(\varepsilon_{\mathrm{m}}+1\right)}, \forall \mathrm{m} \in \mathrm{M},
$$

where $\mathrm{N}_{\mathrm{m}}$ is a random variable representing the number of the packets in the $\mathrm{M} / \mathrm{M} / 1$ queuing system of secondary session $m$.

\subsubsection{CDMA Model}

In the transmission setting as shown in Figure 3.1, multiple primary sessions and secondary sessions share the common channel using CDMA technology.

In the CDMA model, the spread-spectrum bandwidth is denoted by $\mathrm{W}$, the power spectrum density of the Additive White Gaussian Noise (AWGN) is denoted by $\mathrm{N}_{0}$. The channel gain from the sender of link $\mathrm{j}$ to the receiver of link $\mathrm{m}$ is denoted by $g_{\mathrm{jm}}$. The received Bit-Energy-to-Interference-Density Ratio (BEIDR) at the receiver of the secondary session $\mathrm{m}$ is denoted by $\mathrm{y}_{\mathrm{m}}$, which is given by [43]

$$
\mathrm{y}_{\mathrm{m}}=\left(\frac{\mathrm{W}}{\mathrm{R}_{\mathrm{m}}}\right)\left(\frac{\mathrm{g}_{\mathrm{mm}} \mathrm{P}_{\mathrm{m}}}{\delta\left(\sum_{\mathrm{j} \in \mathrm{M}, \mathrm{j} \neq \mathrm{m}} \mathrm{g}_{\mathrm{jm}} \mathrm{P}_{\mathrm{j}}+\sum_{\mathrm{k} \in \mathrm{N}} \mathrm{g}_{\mathrm{km}} \mathrm{P}_{\mathrm{k}}\right)+\mathrm{N}_{0} \mathrm{~W}}\right), \quad \forall \mathrm{m} \in \mathrm{M},
$$

where $\delta$ is the orthogonally factor representing Multiple Access Interference (MAI) from the imperfect orthogonal spreading codes. 
We assume Binary Phase Shift Keying (BPSK) modulation is used in the CDMA system. The Bit Error Rate (BER) at the secondary session $\mathrm{m}$ is given by

$$
\mathrm{e}_{\mathrm{m}}=\mathrm{Q}\left(\sqrt{2 \mathrm{y}_{\mathrm{m}}}\right), \quad \forall \mathrm{m} \in \mathrm{M}
$$

where $\mathrm{Q}(\mathrm{x})$ is a Q-function [44].

If a packet is received in error, it will be dropped at the receiver. We assume that the bit errors occur independently in a packet. Therefore the Packet Loss Rate (PLR) due to transmission errors at the secondary session $\mathrm{m}$ is then given by

$$
\mathrm{P}_{\mathrm{m}}^{\mathrm{e}}=1-\left(1-\mathrm{e}_{\mathrm{m}}\right)^{\mathrm{L}_{\mathrm{m}}}, \forall \mathrm{m} \in \mathrm{M}
$$

where $\mathrm{L}_{\mathrm{m}}$ is the number of bits for the packet at the secondary session $\mathrm{m}$.

\subsubsection{QoS Metrics}

We examine two QoS metrics, the PLR and the queuing delay, at each secondary session. The PLR consists of the congestion PLR $\mathrm{P}_{\mathrm{m}}^{\mathrm{c}}$ due to queue overflow and the transmission PLR $\mathrm{P}_{\mathrm{m}}^{\mathrm{e}}$ due to transmission errors. The PLR of the packets at the secondary session $\mathrm{m}$, denoted by $\mathrm{P}_{\mathrm{m}}^{\mathrm{PLR}}$, is given by

$$
\begin{array}{r}
\mathrm{P}_{\mathrm{m}}^{\mathrm{PLR}}=1-\left(1-\mathrm{P}_{\mathrm{m}}^{\mathrm{c}}\right)\left(1-\mathrm{P}_{\mathrm{m}}^{\mathrm{e}}\right) \\
=1-\left(1-\left(\frac{\tau_{\mathrm{m}}}{\mu_{\mathrm{m}}}\right)^{\left(\varepsilon_{\mathrm{m}}+1\right)}\right)\left(1-\mathrm{Q}\left(\sqrt{2 \mathrm{y}_{\mathrm{m}}}\right)\right)^{\mathrm{L}_{\mathrm{m}}}, \\
\forall \mathrm{m} \in \mathrm{M} .
\end{array}
$$


The queuing delay of a packet is defined as the duration from the time when packet arrives in the $M / M / 1$ queuing system to the time when the packet leaves the $M / M / 1$ queuing system. In the $M / M / 1$ queuing model, the queuing delay $D_{m}$ of the packets at the secondary session $\mathrm{m}$ is given by [42]

$$
\mathrm{D}_{\mathrm{m}}=\frac{1 / \mu_{\mathrm{m}}}{1-\tau_{\mathrm{m}} / \mu_{\mathrm{m}}}, \quad \forall \mathrm{m} \in \mathrm{M}
$$

\subsection{Optimal Resource Allocation}

\subsubsection{Problem Formulation}

We optimize the resource allocation to provide QoS guarantee for video streaming at the secondary sessions, while guaranteeing that the interference from the secondary sessions to the primary sessions should be lower than the tolerable threshold.

The quality of the encoded video at the secondary session $m$ is higher if the source rate $\mathrm{Sm}$ is higher, or equivalently the reciprocal of the source rate, $1 / \mathrm{Sm}$, is lower. Therefore, we set the objective of the resource optimization problem to minimize the weighted sum of the reciprocals of the source rates of all secondary sessions, mathematically expressed by $\mathrm{f}_{\mathrm{obj}}=\sum_{\mathrm{m} \in \mathrm{M}} \frac{\mathrm{k}_{\mathrm{m}}}{\mathrm{s}_{\mathrm{m}}}$, where $\mathrm{k}_{\mathrm{m}}$ denotes the quality weight for the secondary session $\mathrm{m}$, and $\sum_{m \in M} k_{m}=1$. We can enable quality differentiation among the video sessions in the set 
M by assigning different quality weights. The video session with a higher quality weight can be allocated a higher source rate, thus leading to a higher video quality.

The resource optimization problem can be stated as: to minimize the weighted sum of the reciprocals of the source rates of all secondary sessions, by optimizing the source rate, the transmission rate, and the transmission power at each secondary session, subject to the power constraints, the requirements of the congestion PLR, the transmission BER, and tolerable interference at the primary receiving points, respectively. Mathematically, the problem is formulated as follows. 


$$
\begin{aligned}
& \operatorname{minimize}_{(\mathrm{S}, \mathrm{R}, \mathrm{P})} \quad \sum_{\mathrm{m} \in \mathrm{M}} \frac{\mathrm{k}_{\mathrm{m}}}{\mathrm{S}_{\mathrm{m}}} \\
& \text { subject to } Q\left(\sqrt{2 y_{m}}\right) \leq e_{t h}, \quad \forall m \in M \text {, } \\
& \mathrm{y}_{\mathrm{m}}=\left(\frac{\mathrm{W}}{\mathrm{R}_{\mathrm{m}}}\right)\left(\frac{\mathrm{g}_{\mathrm{mm}} \mathrm{P}_{\mathrm{m}}}{\delta\left(\sum_{\mathrm{j} \in \mathrm{M}, \mathrm{j} \neq \mathrm{m}} \mathrm{g}_{\mathrm{jm}} \mathrm{P}_{\mathrm{j}}+\sum_{\mathrm{k} \in \mathrm{N}} \mathrm{g}_{\mathrm{km}} \mathrm{P}_{\mathrm{k}}\right)+\mathrm{N}_{0} \mathrm{~W}}\right), \quad \forall \mathrm{m} \in \mathrm{M}, \\
& \mu_{\mathrm{m}}=\mathrm{R}_{\mathrm{m}} / \mathrm{L}_{\mathrm{m}}, \quad \forall \mathrm{m} \in \mathrm{M} \text {, } \\
& \tau_{\mathrm{m}}=\mathrm{S}_{\mathrm{m}} / \mathrm{L}_{\mathrm{m}}, \quad \forall \mathrm{m} \in \mathrm{M}, \\
& \left(\frac{\tau_{\mathrm{m}}}{\mu_{\mathrm{m}}}\right)^{\left(\varepsilon_{\mathrm{m}}+1\right)} \leq \mathrm{P}_{\mathrm{th}}, \quad \forall \mathrm{m} \in \mathrm{M}, \\
& \tau_{\mathrm{m}} \leq \mu_{\mathrm{m}}, \quad \forall \mathrm{m} \in \mathrm{M}, \\
& \delta \sum_{\mathrm{m} \in \mathrm{M}} \mathrm{g}_{\mathrm{mk}} \mathrm{P}_{\mathrm{m}} \leq \mathrm{I}_{\mathrm{k}}, \quad \forall \mathrm{k} \in \mathrm{N}, \\
& 0 \leq \mathrm{P}_{\mathrm{m}} \leq \mathrm{P}_{\max }, \quad \forall \mathrm{m} \in \mathrm{M} \text {, } \\
& \mathrm{R}_{\mathrm{m}}>0, \quad \forall \mathrm{m} \in \mathrm{M} \text {, } \\
& \mathrm{S}_{\mathrm{m}}>0, \quad \forall \mathrm{m} \in \mathrm{M} \text {, }
\end{aligned}
$$

where $\mathrm{S}$ is the vector of the source rates of all secondary sessions, $\mathrm{R}$ is the vector of the transmission rates of all secondary sessions, $\mathrm{P}$ is the vector of the transmission powers of all secondary sessions, $e_{\text {th }}$ is the threshold of BER, $P_{\text {th }}$ is the threshold of congestion PLR, $\mathrm{P}_{\max }$ is the maximum transmission power at the secondary session, and $\mathrm{I}_{\mathrm{k}}$ is the interference threshold tolerable by the receiving point of the primary session $\mathrm{k}$.

$\mathrm{Q}$ function is a monotonically decreasing function. Therefore we can convert the constraints, $Q\left(\sqrt{2 y_{m}}\right) \leq e_{t h}$ and $y_{m}=\left(\frac{W}{R_{m}}\right)\left(\frac{g_{m m} P_{m}}{\delta\left(\sum_{j \in M, j \neq m} g_{j m} P_{j}+\sum_{k \in N} g_{k m} P_{k}\right)+N_{0} W}\right)$, in the 
optimization problem (11), to an equivalent form $\left(\frac{\mathrm{W}}{\mathrm{R}_{\mathrm{m}}}\right)\left(\frac{\mathrm{g}_{\mathrm{mm}} \mathrm{P}_{\mathrm{m}}}{\delta\left(\sum_{\mathrm{j} \in \mathrm{M}, \mathrm{j} \neq \mathrm{m}} \mathrm{g}_{\mathrm{jm}} \mathrm{P}_{\mathrm{j}}+\sum_{\mathrm{k} \in \mathrm{N}} \mathrm{g}_{\mathrm{km}} \mathrm{P}_{\mathrm{k}}\right)+\mathrm{N}_{0} \mathrm{~W}}\right) \geq \frac{\left(\mathrm{Q}^{-1}\left(\mathrm{e}_{\mathrm{th}}\right)\right)^{2}}{2}$ where $\mathrm{Q}^{-1}(\mathrm{x})$ is the inverse Qfunction [44]. After the conversion, the optimization problem (12) is changed to the following equivalent form:

$$
\begin{aligned}
& \operatorname{minimize}_{(S, R, P)} \quad \sum_{m \in M} \frac{k_{m}}{S_{m}} \\
& \text { subject to }\left(\frac{\mathrm{W}}{\mathrm{R}_{\mathrm{m}}}\right)\left(\frac{\mathrm{g}_{\mathrm{mm}} \mathrm{P}_{\mathrm{m}}}{\delta\left(\sum_{\mathrm{j} \in \mathrm{M}, \mathrm{j} \neq \mathrm{m}} \mathrm{g}_{\mathrm{jm}} \mathrm{P}_{\mathrm{j}}+\sum_{\mathrm{k} \in \mathrm{N}} \mathrm{g}_{\mathrm{km}} \mathrm{P}_{\mathrm{k}}\right)+\mathrm{N}_{\mathrm{o}} \mathrm{W}}\right) \geq \frac{\left(\mathrm{Q}^{-1}\left(\mathrm{e}_{\mathrm{th}}\right)\right)^{2}}{2}, \forall \mathrm{m} \in \mathrm{M} \text {, } \\
& \mu_{\mathrm{m}}=\mathrm{R}_{\mathrm{m}} / \mathrm{L}_{\mathrm{m}}, \quad \forall \mathrm{m} \in \mathrm{M}, \\
& \tau_{\mathrm{m}}=\mathrm{S}_{\mathrm{m}} / \mathrm{L}_{\mathrm{m}}, \quad \forall \mathrm{m} \in \mathrm{M}, \\
& \left(\frac{\tau_{\mathrm{m}}}{\mu_{\mathrm{m}}}\right)^{\left(\varepsilon_{\mathrm{m}}+1\right)} \leq \mathrm{P}_{\mathrm{th}}, \quad \forall \mathrm{m} \in \mathrm{M}, \\
& \tau_{\mathrm{m}} \leq \mu_{\mathrm{m}}, \quad \forall \mathrm{m} \in \mathrm{M}, \\
& \delta \sum_{\mathrm{m} \in \mathrm{M}} \mathrm{g}_{\mathrm{mk}} \mathrm{P}_{\mathrm{m}} \leq \mathrm{I}_{\mathrm{k}}, \quad \forall \mathrm{k} \in \mathrm{N}, \\
& 0 \leq \mathrm{P}_{\mathrm{m}} \leq \mathrm{P}_{\max }, \quad \forall \mathrm{m} \in \mathrm{M}, \\
& \mathrm{R}_{\mathrm{m}}>0, \quad \forall \mathrm{m} \in \mathrm{M} \text {, } \\
& \mathrm{S}_{\mathrm{m}}>0, \quad \forall \mathrm{m} \in \mathrm{M} \text {. }
\end{aligned}
$$

Let $\omega_{\mathrm{th}}=\frac{\left(\mathrm{Q}^{-1}\left(\mathrm{e}_{\mathrm{th}}\right)\right)^{2}}{2}$ represent the threshold of the received BEIDR. If the received BEIDR at the receiver of a secondary session is larger than $\omega_{t h}$, the BER of the secondary session will be less than $\mathrm{e}_{\mathrm{th}}$. 
In the optimization problem (12), the first constraint $\left(\frac{\mathrm{W}}{\mathrm{R}_{\mathrm{m}}}\right)\left(\frac{\mathrm{g}_{\mathrm{mm}} \mathrm{P}_{\mathrm{m}}}{\delta\left(\sum_{\mathrm{j} \in \mathrm{M}, \mathrm{j} \neq \mathrm{m}} \mathrm{g}_{\mathrm{jm}} \mathrm{P}_{\mathrm{j}}+\sum_{\mathrm{k} \in \mathrm{N}} \mathrm{g}_{\mathrm{km}} \mathrm{P}_{\mathrm{k}}\right)+\mathrm{N}_{0} \mathrm{~W}}\right) \geq \frac{\left(\mathrm{Q}^{-1}\left(\mathrm{e}_{\mathrm{th}}\right)\right)^{2}}{2}$, requires the received BEIDR at the receiver of the secondary session $m$ to be no less than the threshold $\omega_{\text {th }}$. The second constraint, $\mu_{m}=R_{m} / L_{m}$, represents packet departure rate out of the $M / M / 1$ queue. The third constraint, $\tau_{m}=S_{m} / L_{m}$, represents packet arrival rate to the $M / M / 1$ queue. The fourth constraint, $\left(\frac{\tau_{\mathrm{m}}}{\mu_{\mathrm{m}}}\right)^{\left(\varepsilon_{\mathrm{m}}+1\right)} \leq \mathrm{P}_{\mathrm{th}}$, requires that the packets at the secondary session $\mathrm{m}$ have a congestion PLR no larger than the threshold $P_{t h}$. The fifth constraint, $\tau_{\mathrm{m}} \leq \mu_{\mathrm{m}}$, requires that the packet arrival rate should be no larger than the packet departure rate at the sender of the secondary session $\mathrm{m}$ in order to maintain a stable $\mathrm{M} / \mathrm{M} / 1$ queuing system.

\subsubsection{Optimal Solution}

We convert the first constraint, $\left(\frac{\mathrm{W}}{\mathrm{R}_{\mathrm{m}}}\right)\left(\frac{\mathrm{gmm}_{\mathrm{m}}}{\delta\left(\sum_{\mathrm{j} \in \mathrm{M}, \mathrm{j} \neq \mathrm{m}} \mathrm{g}_{\mathrm{jm}} \mathrm{P}_{\mathrm{j}}+\sum_{\mathrm{k} \in \mathrm{N}} \mathrm{g}_{\mathrm{km}} \mathrm{P}_{\mathrm{k}}\right)+\mathrm{N}_{0} \mathrm{~W}}\right) \geq \frac{\left(\mathrm{Q}^{-1}\left(\mathrm{e}_{\mathrm{th}}\right)\right)^{2}}{2}$, in the optimization problem (13), to an equivalent form $\frac{\delta \omega_{t h} R_{m}}{g_{m m} P_{m} W} \sum_{j \in M, j \neq m} g_{j m} P_{j}+$ $\frac{\omega_{\text {th }} \mathrm{R}_{\mathrm{m}}\left(\delta \sum_{\mathrm{k} \in \mathrm{N}} \mathrm{g}_{\mathrm{km}} \mathrm{P}_{\mathrm{k}}+\mathrm{N}_{0} \mathrm{~W}\right)}{\mathrm{g}_{\mathrm{mm}} \mathrm{P}_{\mathrm{m}} \mathrm{W}} \leq 1$. The constraint $\left(\frac{\tau_{\mathrm{m}}}{\mu_{\mathrm{m}}}\right)^{\left(\varepsilon_{\mathrm{m}}+1\right)} \leq \mathrm{P}_{\mathrm{th}}$ is equivalent to $P_{t h}^{-1}\left(\frac{S_{m}}{R_{m}}\right)^{\left(\varepsilon_{m}+1\right)} \leq 1$. The constraint, $\tau_{m} \leq \mu_{m}$, is equivalent to $S_{m}\left(R_{m}\right)^{-1} \leq 1$. The constraint, $\delta \sum_{\mathrm{m} \in \mathrm{M}} \mathrm{g}_{\mathrm{mk}} \mathrm{P}_{\mathrm{m}} \leq \mathrm{I}_{\mathrm{k}}$, is equivalent to $\mathrm{I}_{\mathrm{k}}^{-1} \delta \sum_{\mathrm{m} \in \mathrm{M}} \mathrm{g}_{\mathrm{mk}} \mathrm{P}_{\mathrm{m}} \leq 1$. Therefore, the optimization problem (12) is then converted to the following equivalent form:

$$
\operatorname{minimize}_{(\mathrm{S}, \mathrm{R}, \mathrm{P})} \quad \sum_{\mathrm{m} \in \mathrm{M}} \frac{\mathrm{k}_{\mathrm{m}}}{\mathrm{S}_{\mathrm{m}}}
$$




$$
\begin{array}{ll}
\text { subject to } \frac{\delta \omega_{\mathrm{th}} \mathrm{R}_{\mathrm{m}}}{\mathrm{g}_{\mathrm{mm}} \mathrm{P}_{\mathrm{m}} \mathrm{W}} \sum_{\mathrm{j} \in \mathrm{M}, \mathrm{j} \neq \mathrm{m}} g_{\mathrm{jm}} \mathrm{P}_{\mathrm{j}}+\frac{\omega_{\mathrm{th}} \mathrm{R}_{\mathrm{m}}\left(\delta \sum_{\mathrm{k} \in \mathrm{N}} \mathrm{g}_{\mathrm{km}} \mathrm{P}_{\mathrm{k}}+\mathrm{N}_{0} \mathrm{~W}\right)}{\mathrm{g}_{\mathrm{mm}} \mathrm{P}_{\mathrm{m}} \mathrm{W}} \leq 1, & \forall \mathrm{m} \in \mathrm{M}, \\
\mathrm{P}_{\mathrm{th}}^{-1}\left(\frac{\mathrm{S}_{\mathrm{m}}}{\mathrm{R}_{\mathrm{m}}}\right)^{\left(\varepsilon_{\mathrm{m}}+1\right)} \leq 1, & \forall \mathrm{m} \in \mathrm{M}, \\
\mathrm{S}_{\mathrm{m}}\left(\mathrm{R}_{\mathrm{m}}\right)^{-1} \leq 1, & \forall \mathrm{m} \in \mathrm{M}, \\
\mathrm{I}_{\mathrm{k}}^{-1} \delta \sum_{\mathrm{m} \in \mathrm{M} \mathrm{g}_{\mathrm{mk}} \mathrm{P}_{\mathrm{m}} \leq 1,} & \forall \mathrm{k} \in \mathrm{M}, \\
0 \leq \mathrm{P}_{\mathrm{m}} \leq \mathrm{P}_{\mathrm{max}}, & \forall \mathrm{m} \in \mathrm{M}, \\
\mathrm{R}_{\mathrm{m}}>0, & \forall \mathrm{m} \in \mathrm{M} . \\
\mathrm{S}_{\mathrm{m}}>0, & \forall
\end{array}
$$

In the optimization problem (13), the objective function and the left side of the first constraint are polynomials [45], the left sides of the second, third, and fourth constraints are monomials [45]. Therefore the optimization problem is a geometric programming problem [45]. The GP problem can be converted to a convex optimization problem based on a logarithmic change of variables and a logarithmic transformation of the objective and constraint functions [45]. The convex optimization problem can be then solved efficiently using the interior-point methods [46].

The proposed resource optimization algorithm can be performed by the base station. In the initialization stage, the parameters $\left(\mathrm{W}, \delta, \mathrm{N}_{0}\right)$ are obtained by the base station, the parameters $\left(e_{t h}, P_{t h}, P_{\text {max }}\right)$ are set by the administrator, the parameters $\left(L_{m}, \varepsilon_{m}, g_{m j}\right)$ are provided by the sender of the secondary session $\mathrm{m}$, the parameters $\left(\mathrm{P}_{\mathrm{k}}, \mathrm{I}_{\mathrm{k}}\right)$ are provided 
by the sender of the primary session $\mathrm{k}$. The base station collects the parameters, computes the optimal source rate, transmission rate, and transmission power for each secondary session by solving the optimization problem (13), and then feeds them back to the sender of each secondary session, respectively. The sender of each secondary session performs video streaming using the optimal source rate, transmission rate, and transmission power.

\subsection{Simulations}

\subsubsection{Simulation Setting}

We consider a circle area of the CDMA wireless network, which consists of one base station, one primary user, and 4 secondary users. The network setting is shown in Figure. 3.1. The radius of the circle area is $1000 \mathrm{~m}$. The base station is located at the centre, while the primary and secondary users are randomly placed inside the circle area. We assume that there are one primary session and two secondary sessions. The primary session is the uplink connectivity from a primary user to the base station. The two secondary sessions are all single-hop video streaming sessions.

In CDMA model, we set the channel bandwidth $\mathrm{W}=10 \mathrm{MHz}$, the orthogonally factor $\delta=0.1$, and the noise power spectrum density $\mathrm{N}_{0}=10^{-13} \mathrm{~W} / \mathrm{Hz}$. The maximum transmission power is set to $1.0 \mathrm{~W}$ for all secondary sessions. The channel gain from the 
sender of link $\mathrm{j}$ to the receiver of link $\mathrm{m}$ is given by $g_{\mathrm{jm}}=10^{5} / \mathrm{d}_{\mathrm{jm}}^{4}$, where $\mathrm{d}_{\mathrm{jm}}$ is the distance from the sender of link $\mathrm{j}$ to the receiver of link $\mathrm{m}$. We set the interference threshold at the primary receiving point to $10^{-7} \mathrm{~W}$. The transmission power at the primary user is set to $0.5 \mathrm{~W}$. In the $\mathrm{M} / \mathrm{M} / 1$ queuing model, we set the average packet length to 1000 bits, and the queue length to 20 packets, for all secondary sessions. In the setting of QoS thresholds, we set the threshold of transmission BER $e_{\text {th }}=10^{-6}$, the threshold of congestion PLR $\mathrm{P}_{\text {th }}=0.01$. At each video session, the Foreman CIF sequence is encoded using Joint Scalable Video Model (JSVM) video codec [47].

We compare the performances for each secondary session between the proposed scheme, in which the source rate, the transmission rate, and the transmission power for each secondary session are optimized by solving the optimization problem (14), and the uniform scheme, in which the source rate, the transmission rate, and the transmission power are uniformly allocated in two secondary sessions, respectively. In order for fair comparison, the resources consumed in the two schemes are set to the same. In other words, the sum of the source rates, the sum of the transmission rates, and the sum of the transmission powers, of the two secondary sessions, are all equal in the two schemes.

\subsubsection{Simulation Results}


The comparison of the PLR between the two schemes is shown in Figure 3.3. The proposed scheme achieves the same PLR $1.1 \%$ in both secondary sessions. The proposed scheme provides PLR guarantee to all video sessions by optimally utilizing the resources. On the other hand, the uniform scheme does not allocate the resources appropriately, thus causing a high PLR at the secondary session 1.

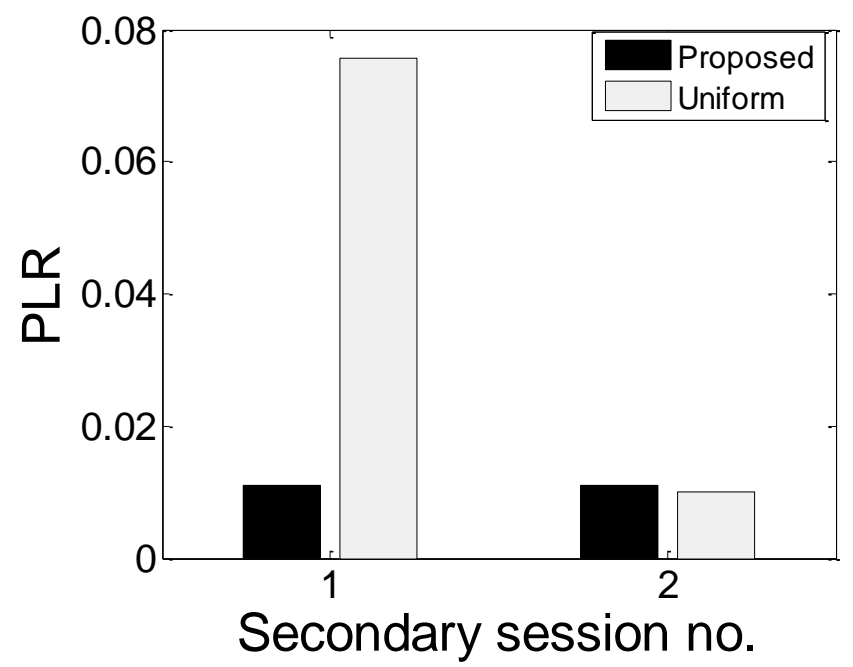

Figure 3.3 Comparison of PLR between the proposed scheme and uniform scheme

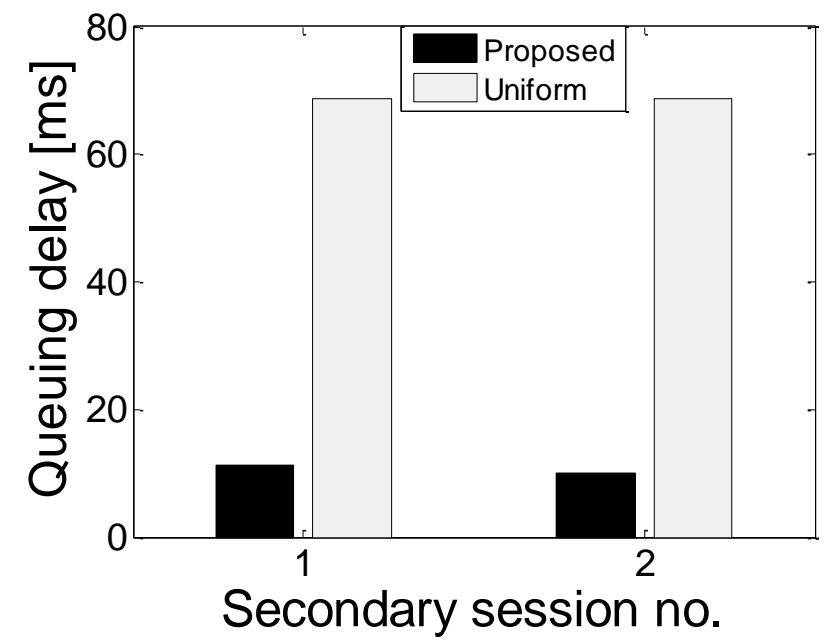

Figure 3.4 Comparison of queuing delay between the proposed scheme and the uniform scheme 
The comparison of the queuing delay between the two schemes is shown in Figure 3.4. The queuing delay in both sessions in the proposed scheme is $10.61 \mathrm{~ms}$, which is much lower than that in the uniform scheme. The proposed scheme can provide queuing delay guarantee to the delay-sensitive video streaming sessions.

The comparison of frame PSNR of the Foreman Common Intermediate Format (CIF) sequence between the proposed scheme and the uniform scheme is shown in Figure 3.5. We do not use any error concealment at the video decoder. Due to the encoding dependency, a lost frame will cause the remaining frames in the same Group of Pictures (GOP) undecodable, which is called error propagation. The Peak Signal to Noise Ratio (PSNR) comparison for the secondary session 1 is shown in Figure 3.5(a), from which we can see much more undecodable frames in the uniform scheme than those in the proposed scheme. This is due to a much higher PLR in the uniform scheme than that in the proposed scheme. The average PSNR in the secondary session 1 is $36.506 \mathrm{~dB}$ in the proposed scheme, and $30.368 \mathrm{~dB}$ in the uniform scheme, respectively. The PSNR comparison for the secondary session 2 is shown in Figure 3.5(b). The proposed scheme and the uniforms scheme have a close source rate and a close PLR in the secondary session 2. Therefore, they have a similar average PSNR. The average PSNR in the 
secondary session 2 is $36.994 \mathrm{~dB}$ in the proposed scheme, and $36.250 \mathrm{~dB}$ in the uniform scheme, respectively.

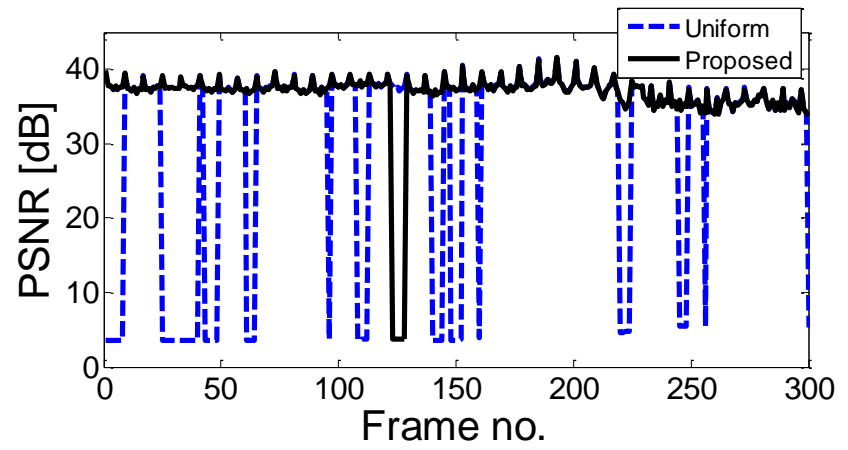

(a)

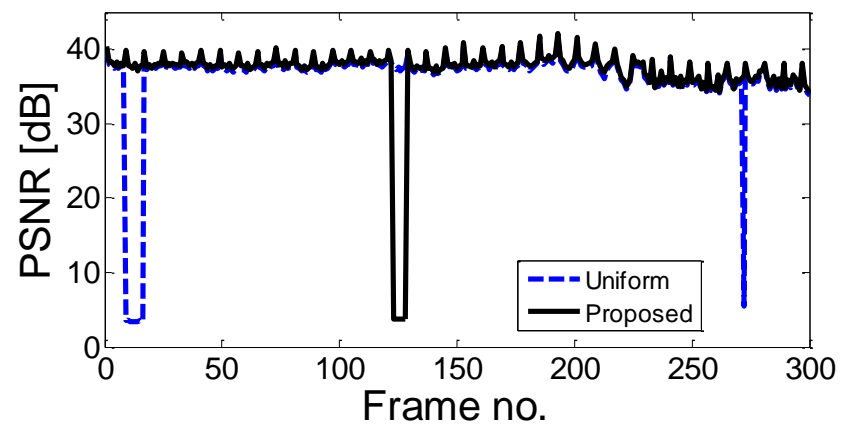

(b)

Figure 3.5 Comparison of frame PSNR of Forman CIF sequence between the proposed scheme and the uniform scheme: (a) secondary session 1 , and (b) secondary session 2

We show the impact of the quality weight in Figure 3.6. The sum of the quality weights of the two secondary sessions is equal to 1 . When the quality weight of the secondary session 1 is increased from 0.05 to 0.95 , the optimal source rate of the secondary session 1 is increased from $144.5 \mathrm{Kbps}$ to $585.9 \mathrm{Kbps}$, while the optimal source rate of the secondary session 2 is decreased from $615.5 \mathrm{Kbps}$ to $201.5 \mathrm{Kbps}$, as shown in Figure 3.6. A higher source rate leads to a higher quality of the encoded video. 
Therefore, the video quality of a video session can be adjusted by changing the corresponding quality weight.

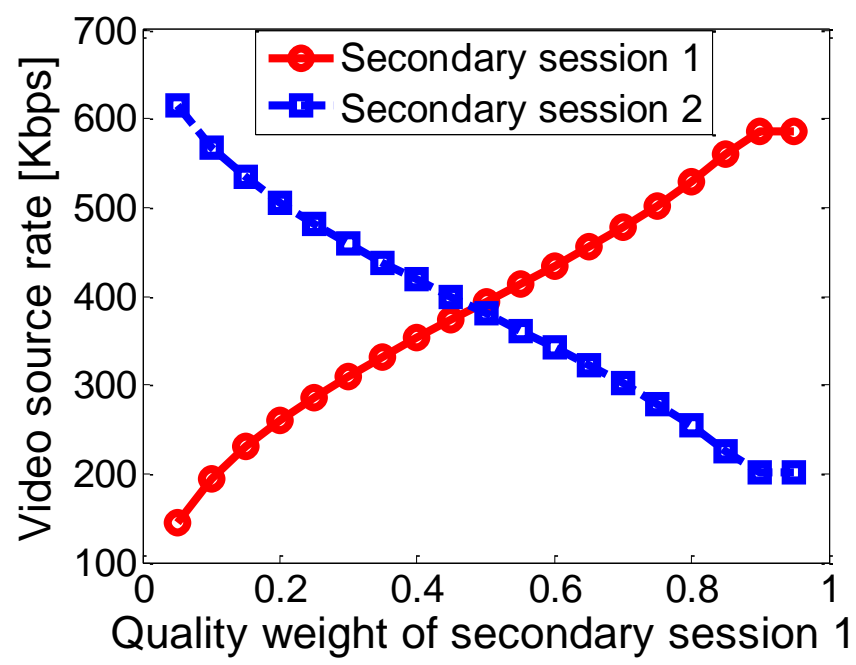

Figure 3.6 Variation of video source rates with the quality weight of secondary session 1

\subsection{Chapter Summary}

In this chapter, we optimized the resource allocation to provide QoS guarantee for video streaming over spectrum underlay cognitive radio networks. Specifically, we formulated the resource allocation problem into an optimization problem, which jointly optimizes the source rate, the transmission rate, and the transmission power at each secondary session to minimize the weighted sum of the reciprocals of the source rates of all secondary sessions. The optimization problem is formulated into a GP problem, which can be converted to a convex optimization problem and then be solved efficiently. In the simulations, we demonstrated that the proposed scheme can provide QoS guarantee, thus 
achieving a higher video quality for the video streaming sessions, compared to the uniform scheme. 


\section{Chapter 4}

\section{Multi-Layered Video Streaming over Multi-Channel CR Networks}

IN multi-channel cognitive radio networks, each secondary user can use multiple channels for data transmission to improve the spectrum utilization. In this chapter, we investigate the resource allocation problem for multi-layered video streaming over multichannel cognitive radio networks. The video is encoded into multiple layers, each of which is delivered over a separate channel. Moreover, we jointly optimize the source rate, the transmission rate, and the transmission power at each video session in each channel to provide Quality of Service (QoS) guarantee to all video sessions in the secondary network.

\subsection{Problem Statement}

We investigate the resource allocation problem for multi-layered video streaming over multi-channel cognitive radio networks. It is challenging to provide QoS guarantee for video streaming over multi-channel spectrum underlay cognitive radio networks. First, the QoS supports depend on the resource allocation at each user and in each channel, 
which is a nontrivial task. Second, the interferences among the users may corrupt the video packets, thus degrading the video quality. Third, the resource allocation to the secondary users needs to be controlled appropriately to guarantee undisturbed operation of primary users. In this scheme, the video is encoded into multiple layers, each of which is delivered over a separate channel. Moreover, we jointly optimize the source rate, the transmission rate, and the transmission power at each video session in each channel to provide QoS guarantee to all video sessions in the secondary network. The optimization problem is formulated into a Geometric Programming (GP) problem, which can be solved efficiently.

\subsection{Multi-Channel CR System Models}

\subsubsection{Network Topology}

We consider video streaming over multi-channel spectrum underlay cognitive radio networks. The set of the secondary sessions is denoted by $\mathrm{M}$, and the set of the primary sessions is denoted by $\mathrm{K}$. We assume that all the secondary sessions in the set $\mathrm{M}$ are single-hop video streaming sessions. The set of the available channels is denoted by $\mathrm{N}$. The set of the video layers for a video is denoted by H. Each video streaming session can use multiple channels in the channel set $\mathrm{N}$. 


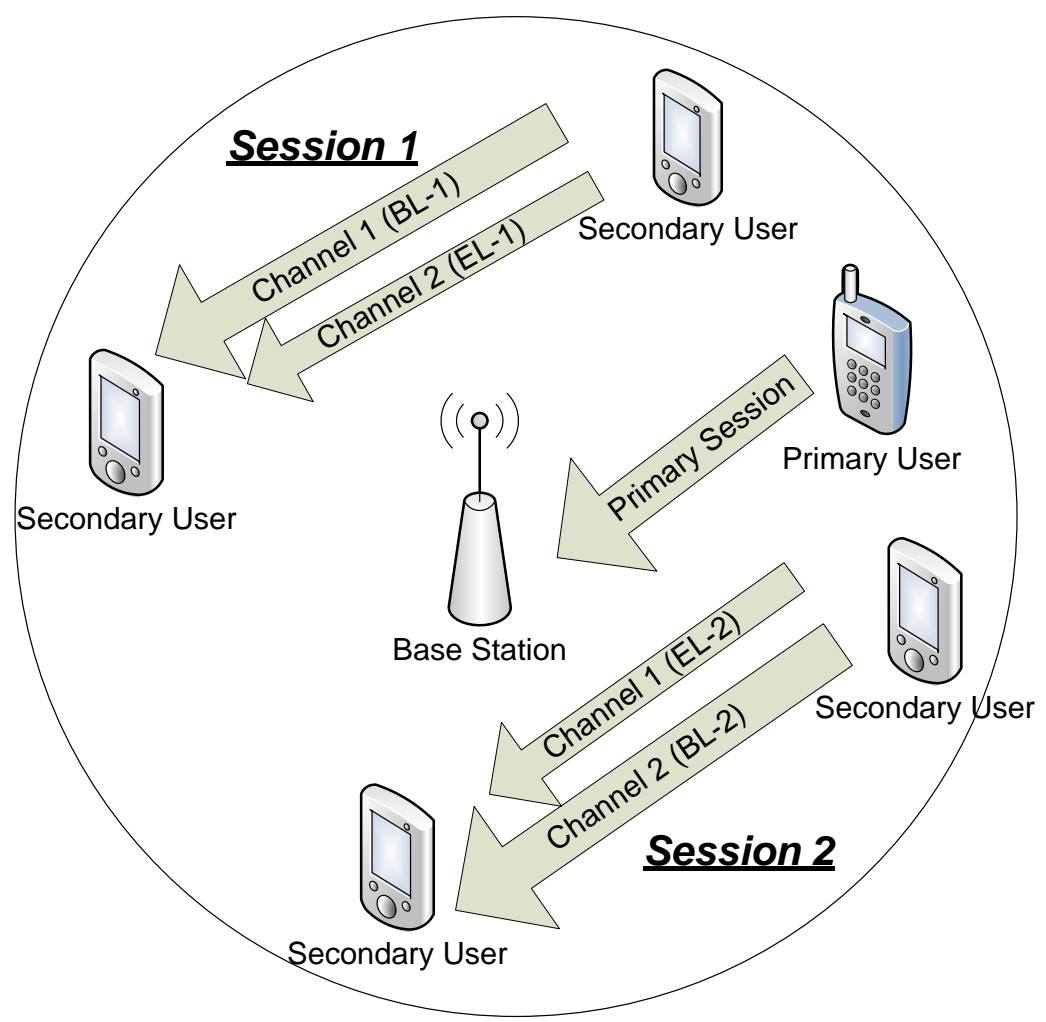

Figure 4.1 Illustration of multi-layered video streaming over multi-channel cognitive radio networks

To better describe the proposed approach, we set $\mathrm{H}=\mathrm{N}=\{1,2\}$, which means that the number of the layers for a video and the number of the available channels are both set to 2. The video is encoded into a Base Layer (BL) and an Enhancement Layer (EL). We employ a channel selection scheme as follows. All the video sessions in the set $\mathrm{M}$ is ordered based on the start time. The video sessions with an odd order, called odd video sessions, choose channel 1 to deliver the base layer and channel 2 to deliver the enhancement layer. The video sessions with an even order, called even video sessions, choose channel 1 to deliver the enhancement layer and channel 2 to deliver the base layer. Since the base layer has more stringent QoS requirements, it demands a larger portion of 
resource than the enhancement layer. The employed channel selection scheme balances the number of the BLs and ELs across channels, thus balancing the resource allocation.

Figure 4.1 illustrates the scenario of multi-layered video streaming over multi-channel cognitive radio networks. Though we study the specific scenario with two video layers and two channels in this thesis, the proposed approach can be easily applied to the generic scenario with a larger number of video layers and channels.

\subsubsection{Rate Distortion Model for Layered Video}

A video is encoded to a base layer and an enhancement layer. The rate distortion relationship of video $\mathrm{m}$ is modeled by

$$
\mathrm{d}_{\mathrm{m}}=\left(\frac{\varphi_{\mathrm{m} 1}}{\mathrm{~s}_{\mathrm{m} 1}{ }{ }_{m}}+\varphi_{\mathrm{m} 2}\right) \frac{1}{\mathrm{~s}_{\mathrm{m} 2} \beta_{m}}, \quad \forall \mathrm{m} \in \mathrm{M}
$$

where $d_{m}$ is the encoding distortion of video $m, S_{m 1}$ is the bit rate of the base layer of video $\mathrm{m}, \mathrm{S}_{\mathrm{m} 2}$ is the bit rate of the enhancement layer of video $\mathrm{m}$, and $\varphi_{\mathrm{m} 1}, \varphi_{\mathrm{m} 2}, \alpha_{m}$, and $\beta_{m}$ are the model parameters. The bit rates and the model parameters in Equation (14) are all positive real number. The model parameters can be found using the data fitting technique. 
Table 4.1 Comparison between actual distortion and estimated distortion for FORMAN CIF sequence

\begin{tabular}{|l|l|l|l|}
\hline Bit Rate of Base Layer [Kbps] & 170.896 & 138.496 & 109.567 \\
\hline Bit Rate of Enhancement Layer [Kbps] & 453.232 & 361.416 & 284.928 \\
\hline Actual Distortion & 16.081 & 20.817 & 27.673 \\
\hline Estimated Distortion & 17.009 & 20.916 & 26.016 \\
\hline
\end{tabular}

We use Joint Scalable Video Model (JSVM) codec [7] to encode the Foreman CIF sequence to get three sets of bit rates and distortions, with which we find the model parameters by minimizing the mean absolute error between the estimated distortion and the actual distortion. The obtained model parameters for Foreman CIF sequence are: $\varphi_{\mathrm{m} 1}=6329.3, \varphi_{\mathrm{m} 2}=4119.8, \alpha=0.90$, and $\beta=0.90$. The comparison between actual distortion and estimated distortion is given in Table 4.1. The mean absolute error obtained from Table I is 0.8947, which demonstrates that Equation (1) can closely represent the actual rate distortion relationship.

\subsubsection{Queuing Model}

At video session $\mathrm{m}$, the sender encodes the video into a base layer and an enhancement layer, each of which is buffered in an independent M/M/1 queue [42] before transmitted to the receiver over a separate channel. Let $L_{m n}$ denote the average length of the packets, $\mathrm{S}_{\mathrm{mn}}$ denote the source rate, $\mathrm{R}_{\mathrm{mn}}$ denote the transmission rate, at video session $\mathrm{m}$ in channel $\mathrm{n}$. The packet arrival rate to the $\mathrm{M} / \mathrm{M} / 1$ queue at video session $\mathrm{m}$ in channel $\mathrm{n}$ is 
given by $\tau_{\mathrm{mn}}=\mathrm{S}_{\mathrm{mn}} / \mathrm{L}_{\mathrm{mn}}$, and the packet departure rate out of the M/M/1 queue at video session $m$ in channel $n$ is given by $\mu_{m n}=R_{m n} / L_{m n}$. The $M / M / 1$ queue needs to satisfy the follow condition in order to be stable [42]: $\tau_{\mathrm{mn}} \leq \mu_{\mathrm{mn}}, \forall \mathrm{m} \in \mathrm{M}, \mathrm{n} \in \mathrm{N}$.

We use tail probability of M/M/1 queuing system to represent the congestion PLR. Let the threshold $\varepsilon_{\mathrm{mn}}$ represent the buffer length at video session $\mathrm{m}$ in channel $\mathrm{n}$. The tail probability $\mathrm{P}_{\mathrm{mn}}^{\mathrm{c}}$ at video session $\mathrm{m}$ in channel $\mathrm{n}$ is given by [42]

$$
\mathrm{P}_{\mathrm{mn}}^{\mathrm{c}}=\mathrm{P}_{\mathrm{r}}\left(\mathrm{N}_{\mathrm{mn}}>\varepsilon_{\mathrm{mn}}\right)=\left(\frac{\tau_{\mathrm{mn}}}{\mu_{\mathrm{mn}}}\right)^{\left(\varepsilon_{\mathrm{mn}}+1\right)}, \forall \mathrm{m} \in \mathrm{M}, \mathrm{n} \in \mathrm{N},
$$

where $\mathrm{N}_{\mathrm{mn}}$ is a random variable representing the number of the packets in the $\mathrm{M} / \mathrm{M} / 1$ queuing system at video session $\mathrm{m}$ in channel $\mathrm{n}$.

\subsubsection{Transmission Model}

For video streaming over multi-channel cognitive radio networks, multiple video sessions share the resource in an individual channel. We assume that each channel has the same bandwidth denoted by W. The power spectrum density of the Additive White Gaussian Noise (AWGN) is denoted by $\mathrm{N}_{0}$. The channel gain from the sender of link $\mathrm{j}$ to the receiver of link $m$ is denoted by $g_{j m}$. The received Bit-Energy-to-Interference-Density Ratio (BEIDR) at the receiver of the video session $m$ in channel $n$ is denoted by $y_{m n}$, which is given by [9]

$$
\mathrm{y}_{\mathrm{mn}}=\left(\frac{\mathrm{w}}{\mathrm{R}_{\mathrm{mn}}}\right)\left(\frac{\mathrm{g}_{\mathrm{mm}} \mathrm{P}_{\mathrm{mn}}}{\delta\left(\sum_{\mathrm{j} \in \mathrm{M}, \mathrm{j} \neq \mathrm{m}} \mathrm{g}_{\mathrm{j} m} \mathrm{P}_{\mathrm{jn}}+\sum_{\mathrm{k} \in \mathrm{K}} \mathrm{g}_{\mathrm{km}} \mathrm{P}_{\mathrm{kn}}\right)+\mathrm{N}_{0} \mathrm{~W}}\right), \quad \forall \mathrm{m} \in \mathrm{M}, \mathrm{n} \in \mathrm{N},
$$


where $\mathrm{P}_{\mathrm{mn}}$ is the transmission power at video session $\mathrm{m}$ in channel $\mathrm{n}$, and $\delta$ is the orthogonal factor representing Multiple Access Interference (MAI) from the imperfect orthogonal spreading codes.

We assume Binary Phase Shift Keying (BPSK) modulation is used in the transmission system. The Bit Error Rate (BER) at video session $\mathrm{m}$ in channel $\mathrm{n}$ is given by $\mathrm{e}_{\mathrm{mn}}=$ $\mathrm{Q}\left(\sqrt{2 \mathrm{y}_{\mathrm{mn}}}\right)$ where $\mathrm{Q}(\mathrm{x})$ is a $\mathrm{Q}$-function.

The Packet Loss Rate (PLR) due to transmission errors at video session $\mathrm{m}$ in channel $\mathrm{n}$ is given by $P_{m n}^{e}=1-\left(1-e_{m n}\right)^{L_{m n}}$, where $L_{m n}$ is the number of bits of the packet at video session $m$ in channel $n$.

The overall PLR consists of two components: 1) the congestion PLR due to queue overflow, and 2) the transmission PLR due to transmission errors. The overall PLR at video session $m$ in channel $n$ is given by $\mathrm{P}_{\mathrm{mn}}^{\mathrm{PLR}}=1-\left(1-\mathrm{P}_{\mathrm{mn}}^{\mathrm{c}}\right)\left(1-\mathrm{P}_{\mathrm{mn}}^{\mathrm{e}}\right)$.

\subsection{Optimal Resource Allocation}

\subsubsection{Problem Formulation}

We optimize the resource allocation to provide QoS guarantee to the video sessions in the secondary network, while protecting the primary users against the excessive interference from the secondary users. The resource optimization problem can be stated 
as to minimize the sum of the encoding distortions of all video sessions by optimizing the source rate, the transmission rate, and the transmission power of each video session in each channel, subject to the power constraints, the requirements of the congestion PLR, the transmission BER, and tolerable interference at the primary receiving points, respectively. Mathematically, the problem is formulated as follows. 


$$
\begin{aligned}
& \text { minimize } \quad \sum_{\mathrm{m} \in \mathrm{M}}\left(\frac{\varphi_{\mathrm{m} 1}}{\mathrm{~S}_{\mathrm{mb}}{ }^{\alpha_{m}}}+\varphi_{\mathrm{m} 2}\right) \frac{1}{\mathrm{~S}_{\mathrm{me}} \beta_{m}} \\
& \text { subject to } Q\left(\sqrt{2 y_{\mathrm{mn}}}\right) \leq \mathrm{e}_{\text {th }}^{(\mathrm{z})}, \quad \forall \mathrm{m} \in \mathrm{M}, \mathrm{n} \in \mathrm{N} \text {, } \\
& \mathrm{y}_{\mathrm{mn}}=\left(\frac{\mathrm{W}}{\mathrm{R}_{\mathrm{mn}}}\right)\left(\frac{\mathrm{g}_{\mathrm{mm}} \mathrm{P}_{\mathrm{mn}}}{\delta\left(\sum_{\mathrm{j} \in \mathrm{M}, \mathrm{j} \neq \mathrm{m}} \mathrm{g}_{\mathrm{jm}} \mathrm{P}_{\mathrm{jn}}+\sum_{\mathrm{k} \in \mathrm{K}} \mathrm{g}_{\mathrm{km}} \mathrm{P}_{\mathrm{kn}}\right)+\mathrm{N}_{0} \mathrm{~W}}\right), \forall \mathrm{m} \in \mathrm{M}, \mathrm{n} \in \mathrm{N}, \\
& \mu_{\mathrm{mn}}=\mathrm{R}_{\mathrm{mn}} / \mathrm{L}_{\mathrm{mn}}, \quad \forall \mathrm{m} \in \mathrm{M}, \mathrm{n} \in \mathrm{N}, \\
& \tau_{\mathrm{mn}}=\mathrm{S}_{\mathrm{mn}} / \mathrm{L}_{\mathrm{mn}}, \quad \forall \mathrm{m} \in \mathrm{M}, \mathrm{n} \in \mathrm{N}, \\
& \left(\frac{\tau_{\mathrm{mn}}}{\mu_{\mathrm{mn}}}\right)^{\left(\varepsilon_{\mathrm{mn}}+1\right)} \leq \mathrm{P}_{\mathrm{th}}^{(\mathrm{z})}, \quad \forall \mathrm{m} \in \mathrm{M}, \mathrm{n} \in \mathrm{N}, \\
& \tau_{\mathrm{mn}} \leq \mu_{\mathrm{mn}}, \quad \forall \mathrm{m} \in \mathrm{M}, \mathrm{n} \in \mathrm{N}, \\
& \delta \sum_{\mathrm{m} \in \mathrm{M}} \mathrm{g}_{\mathrm{mk}} \mathrm{P}_{\mathrm{mn}} \leq \mathrm{I}_{\mathrm{k}}, \quad \forall \mathrm{k} \in \mathrm{K}, \mathrm{n} \in \mathrm{N}, \\
& 0 \leq \mathrm{P}_{\mathrm{mn}} \leq \mathrm{P}_{\max }, \quad \forall \mathrm{m} \in \mathrm{M}, \mathrm{n} \in \mathrm{N} \\
& \mathrm{R}_{\mathrm{mn}}>0, \quad \forall \mathrm{m} \in \mathrm{M}, \mathrm{n} \in \mathrm{N}, \\
& \mathrm{S}_{\mathrm{mn}}>0, \quad \forall \mathrm{m} \in \mathrm{M}, \mathrm{n} \in \mathrm{N},
\end{aligned}
$$

In the optimization problem (17), $S_{\mathrm{mb}}$ and $S_{\text {me }}$ represent the source rates of the base layer and the enhancement layer, respectively, at video session $\mathrm{m}$, the subscripts $\mathrm{b}$ and e represent the channel no., which can be either 1 or 2 , depending on the channel assignment for the video session $\mathrm{m}$. $\mathrm{e}_{\mathrm{th}}^{(\mathrm{z})}$ is the threshold of BER. If the base layer is delivered over the channel, $\mathrm{z}$ is set to 1 , if the enhancement layer is delivered over the channel, $\mathrm{z}$ is set to 2 . The same setting is applied for the superscript in $\mathrm{P}_{\mathrm{th}}^{(\mathrm{z})}$, the threshold of congestion PLR. Since the base layer requires a more stringent QoS than the 
enhancement layer, we set $\mathrm{e}_{\mathrm{th}}^{(1)}<\mathrm{e}_{\mathrm{th}}^{(2)}$ and $\mathrm{P}_{\mathrm{th}}^{(1)}<\mathrm{P}_{\mathrm{th}}^{(2)}$. $\mathrm{P}_{\max }$ is the maximum transmission power for all video sessions, and $\mathrm{I}_{\mathrm{k}}$ is the interference threshold tolerable by the receiving point of the primary session $\mathrm{k}$.

\subsubsection{Optimal Solution}

The optimization problem (17) can be converted to the following equivalent problem:

$$
\begin{aligned}
& \text { minimize } \quad \sum_{\mathrm{m} \in \mathrm{M}}\left(\frac{\varphi_{\mathrm{m} 1}}{\mathrm{~S}_{\mathrm{mb}}{ }^{\alpha_{m}}}+\varphi_{\mathrm{m} 2}\right) \frac{1}{\mathrm{~S}_{\mathrm{me}} \beta_{m}} \\
& \text { subject to } \frac{\delta \omega_{t h} R_{m n}}{g_{m m} P_{m n} W} \sum_{j \in M, j \neq m} g_{j m} P_{j n}+\frac{\omega_{t h} R_{m n}\left(\delta \sum_{k \in K} g_{k m} P_{k n}+N_{0} W\right)}{g_{m m} P_{m n} W} \leq 1 \text {, } \\
& \forall \mathrm{m} \in \mathrm{M}, \mathrm{n} \in \mathrm{N} \\
& \left(\frac{S_{\mathrm{mn}}}{\mathrm{R}_{\mathrm{mn}}}\right)^{\left(\varepsilon_{\mathrm{mn}}+1\right)} / \mathrm{P}_{\mathrm{th}}^{(\mathrm{z})} \leq 1 \\
& \forall \mathrm{m} \in \mathrm{M}, \mathrm{n} \in \mathrm{N}, \\
& \mathrm{S}_{\mathrm{mn}} / \mathrm{R}_{\mathrm{mn}} \leq 1, \\
& \forall \mathrm{m} \in \mathrm{M}, \mathrm{n} \in \mathrm{N}, \\
& \mathrm{I}_{\mathrm{k}}^{-1} \delta \sum_{\mathrm{m} \in \mathrm{M}} \mathrm{g}_{\mathrm{mk}} \mathrm{P}_{\mathrm{mn}} \leq 1, \\
& \forall \mathrm{k} \in \mathrm{K}, \mathrm{n} \in \mathrm{N}, \\
& 0 \leq \mathrm{P}_{\mathrm{mn}} \leq \mathrm{P}_{\max }, \\
& \forall \mathrm{m} \in \mathrm{M}, \mathrm{n} \in \mathrm{N}, \\
& \mathrm{R}_{\mathrm{mn}}>0 \text {, } \\
& \forall \mathrm{m} \in \mathrm{M}, \mathrm{n} \in \mathrm{N}, \\
& \mathrm{S}_{\mathrm{mn}}>0, \\
& \forall \mathrm{m} \in \mathrm{M}, \mathrm{n} \in \mathrm{N},
\end{aligned}
$$

where $\omega_{\mathrm{th}}=\frac{1}{2}\left(\mathrm{Q}^{-1}\left(\mathrm{e}_{\mathrm{th}}^{(\mathrm{z})}\right)\right)^{2}$, in which $\mathrm{Q}^{-1}(\mathrm{x})$ is the inverse $\mathrm{Q}$-function .

The optimization problem (5) is a GP problem [45]. The GP problem can be transformed to a convex optimization problem based on a logarithmic change of variables and a logarithmic transformation of the objective and constraint functions [45]. The 
convex optimization problem can be then solved efficiently using the interior-point methods [46].

\subsection{Simulations}

\subsubsection{Simulation Setting}

We consider a wireless network with two orthogonal channels. The network deployment is shown in Figure 4.1. The radius of the circle area is $1500 \mathrm{~m}$. The base station is located at the center, while the primary and secondary users are randomly placed inside the circle area. We assume that there are one primary session and two secondary sessions. The primary session is the uplink connectivity from a primary user to the base station. The two secondary sessions are all single-hop video streaming sessions. Each video session consists of a base layer stream and an enhancement layer stream, each of which is delivered over a separate channel. We set the channel bandwidth $\mathrm{W}=10 \mathrm{MHz}$, the orthogonal factor $\delta=0.1$, and the noise power spectrum density $\mathrm{N}_{0}=10^{-13} \mathrm{~W} / \mathrm{Hz}$. The maximum transmission power is set to $1.0 \mathrm{~W}$. The channel gain from the sender of link $\mathrm{j}$ to the receiver of link $\mathrm{m}$ is given by $\mathrm{g}_{\mathrm{jm}}=10^{5} / \mathrm{d}_{\mathrm{jm}}^{4}$, where $\mathrm{d}_{\mathrm{jm}}$ is the distance from the sender of link $\mathrm{j}$ to the receiver of link $\mathrm{m}$. We set the interference threshold at the primary

receiving point to $10^{-7} \mathrm{~W}$. The transmission power at the primary user is set to $0.5 \mathrm{~W}$. In 
the M/M/1 queuing model, we set the average packet length to 1000 bits, and the buffer length to 20 packets, for all video sessions. We set the BER threshold $\mathrm{e}_{\mathrm{th}}^{(1)}=10^{-6}$ for the base layer, and $\mathrm{e}_{\mathrm{th}}^{(2)}=5 \times 10^{-6}$ for the enhancement layer. We set the threshold of

congestion PLR $\mathrm{P}_{\mathrm{th}}^{(1)}=0.004$ for the base layer, and $\mathrm{P}_{\mathrm{th}}^{(2)}=0.03$ for the enhancement layer. At each video session, the Foreman CIF sequence is encoded using JSVM video codec [47].

\subsubsection{Simulation Results}

We compare the performance between the optimal scheme, in which the source rate, the transmission rate, and the transmission power for each video session are optimized by solving the optimization problem (5), and the equal scheme, in which the source rate, the transmission rate, and the transmission power are equally allocated in two video sessions in each channel, respectively. In order for fair comparison, the total amount of resources in the two schemes is set to be equal. 


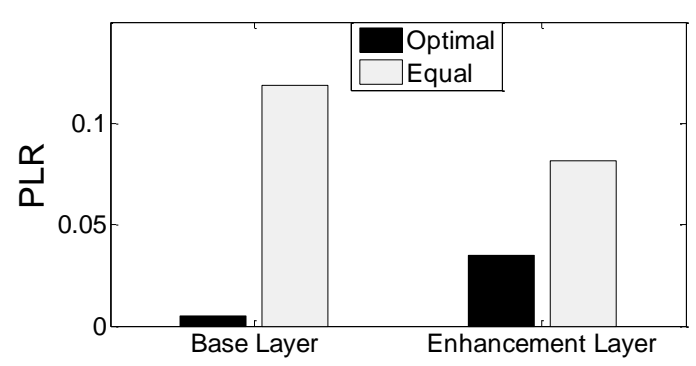

(a)

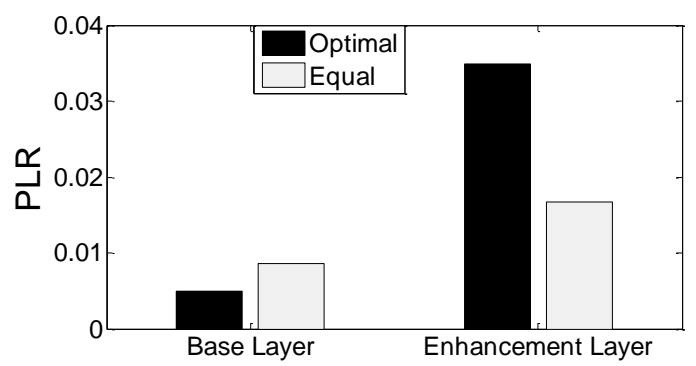

(b)

Figure 4.2 Comparison of PLR between the optimal scheme and the equal scheme: (a) video session 1, and (b) video session 2

The PLR comparison between the two schemes is shown in Figure 4.2. The proposed optimal scheme achieves the low PLRs, $0.49 \%$ for the base layer and $3.48 \%$ for the enhancement layer, respectively, in both video sessions. The proposed scheme provides PLR guarantee to all video sessions by optimally utilizing the resource. On the other hand, the equal scheme does not allocate the resource appropriately, thus causing much higher PLRs in video session 1. 

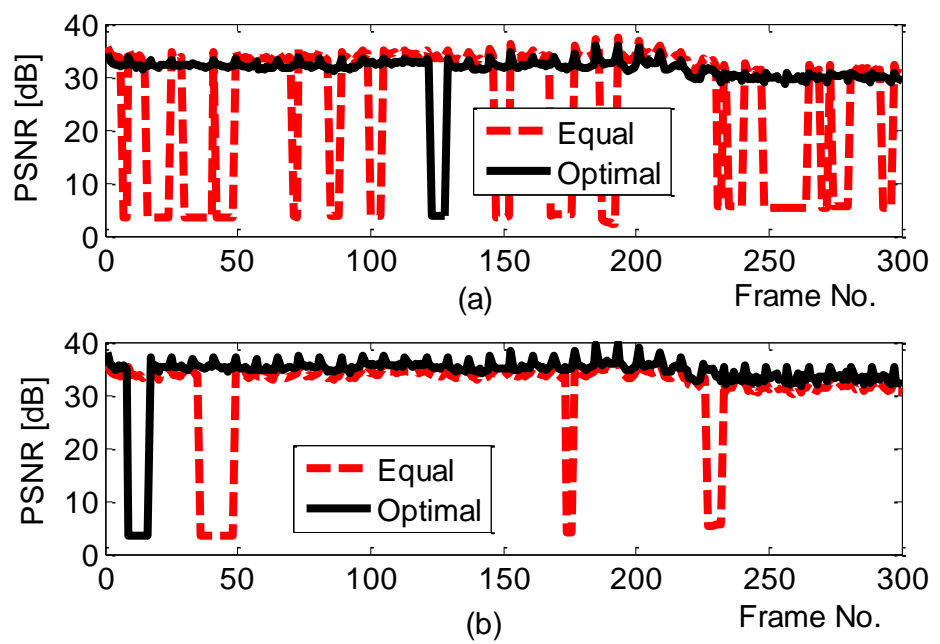

Figure 4.3 Comparison of frame PSNR of Forman CIF sequence between the optimal scheme and equal scheme: (a) video session 1, and (b) video session 2

The comparison of Peak Signal-to-Noise Ratio (PSNR) of the Foreman CIF sequence in the two video sessions between the proposed optimal scheme and the equal scheme is shown in Figure 4.3. We do not use any error concealment at the video decoder. The PSNR comparison for video session 1 is shown in Figure 4.3(a), from which we can see much more undecodable frames in the equal scheme than those in the optimal scheme. This is due to much higher PLRs in the equal scheme. The average PSNR for video session 1 is $31.133 \mathrm{~dB}$ in the proposed optimal scheme and $21.793 \mathrm{~dB}$ in the equal scheme, respectively. The PSNR comparison for video session 2 is shown in Figure 4.3(b). The proposed optimal scheme has higher source rates for both base layer and enhancement layer, and a lower PLR for base layer, thus obtaining an average PSNR of 
$34.098 \mathrm{~dB}$ for video session 2, compared to the average PSNR of $31.193 \mathrm{~dB}$ in the equal scheme.

\subsection{Chapter Summary}

In this chapter, we investigated the resource allocation problem for multi-layered video streaming over multi-channel cognitive radio networks. Specifically, we jointly optimized the source rate, the transmission rate, and the transmission power at each video session in each channel to provide QoS guarantee to all video sessions in the secondary network. The resource allocation problem is formulated into a GP problem, which can be solved efficiently. In the simulations, we demonstrated that the proposed optimal scheme can achieve a lower average PLR, thus leading to a higher average PSNR compared to the equal scheme. 


\section{Chapter 5}

\section{Conclusion and Future Work}

\subsection{Conclusion}

Cognitive radio networks will provide reliable wireless service to mobile users via heterogeneous wireless architectures and dynamic spectrum access techniques. The Quality of Service (QoS) for cognitive radio (CR) networks is an important problem but has received relatively limited attention from the CR community. In this thesis, we propose to efficiently provision the network resource among secondary users according to their QoS requirement for specific multimedia services. To the end, we develop an optimal resource allocation framework for video streaming over the spectrum underlay cognitive radio network. The proposed framework optimizes the secondary users' resource allocation for video streaming service in terms of the tolerance to the network dynamics. We investigate the resource allocation problem for both single layered and multi-layered video streaming over single channel and multi-channel cognitive radio networks respectively. Specifically, we jointly optimize the source rate, the transmission 
rate, and the transmission power at each video session in each channel to provide QoS guarantee to all video sessions in the secondary network. The resource allocation problem is formulated into a GP problem, which can be solved efficiently. In the simulations, we demonstrate that the proposed optimal scheme can achieve a lower average PLR, thus leading to a higher average PSNR compared to the equal scheme.

\subsection{Future Work}

For the future work, we intend to further investigate the CR video streaming system in multi-hop CR resource allocation optimization: the current framework is based on the single-hop CR architecture. However, such system is not compatible to cover the service

for the larger scale network, such as Metropolitan Area Networks. In the future, we intend to device the multi-hop CR resource allocation scheme by allowing secondary users to distributedly select path to transmit according to their QoS requirements. Without the single-hop barrier, the resulting resource allocation scheme would thus be more flexible and scalable. 


\section{Bibliography}

[1] FCC, Spectrum police task force report, FCC 02-155, Nov. 2002.

[2] I. F. Akyildiz, W. Y. Lee, M. C. Vuran, and S. Mohanty, "NeXt generation/dynamic spectrum access/cognitive radio wireless networks: A Survey," Computer Networks, vol. 50, no. 13, pp. 21272159, 2006.

[3] P. J. Kolodzy, "Dynamic spectrum policies: promises and challenges," CommLaw Conspectus, vol. 12, p. 147, 2004.

[4] FCC, “ET docket no 03-222," Notice of Proposed Rule Making and Order, Dec. 2003.

[5] S. Haykin, "Cognitive Radio: Brain-Empowered Wireless Communications," IEEE J. Select Areas in Commun., vol. 23, no. 2, pp. 201-220, Feb. 2005.

[6] L. Giupponi, C. Ibars, "Distributed Cooperation in Cognitive Radio Networks: Overlay Versus Underlay Paradigm," in Pro. of IEEE VTC, 2009.

[7] Q. Zhao, B.M Sadler, “A Survey of dynamic spectrum access: Signal processing, networking and regulatory policy,” IEEE Signal Processing Mag., pp.79-89, May 2007.

[8] FCC Notice of Inquiry and Notice of Proposed Rule Making, "In the matter of establishment of an interference temperature metric to quantify and manage interference and to expand available unlicensed operation in certain fixed, mobile and satellite frequency bands," ET Docket No. 03-237, Nov. 2003. 
[9] J. Hamilla, J. Stern, "Technology Solution to the Spectrum Crisis," Wireless Communication Alliance Symposium, Feb. 2010.

[10] J. Mitola, Cognitive Radio, licentiate thesis, Royal Inst. Of Technol., KTH, Sept. 1999.

[11] Agilent Technologies, "IPTV QoE: Understanding and interpreting MDI values," white paper, 2006.

[12] H. Jiang, W. Zhang, X. Shen, Q. Bi, "Quality of Service Provisioning and Efficient Resource Utilization in CDMA Cellular Communications," IEEE J. Select Area in Commun., vol. 24, no. 1, pp. 4-15, Jan. 2006.

[13] A. Vogel, B. Kerherve, G. V. Bochmann, and J. Gecsei, "Distributed multimedia and QoS: a survey", IEEE Multimedia, vol. 2, no. 2, pp. 10-19, 1995.

[14] L. Le and E. Hossain, "Resource Allocation for Spectrum Underlay in Cognitive Radio Networks," IEEE Transactions on Wireless Communications, vol. 7, no. 12, pp.5306-5315, Dec. 2008.

[15] L. Akter and B. Natarajan, "Distributed Approach for Power and Rate Allocation to Secondary Users in Cognitive Radio Networks," IEEE Trans. on Vehicular Tech., vol. 60, no. 4, pp. 1526-1538, May 2011.

[16] Hu and S. Mao, "Streaming Scalable Videos over Multi-Hop Cognitive Radio Networks," IEEE Transactions on Wireless Communications, vol. 9, no. 11, pp. 3501-3511, Nov. 2010.

[17] S. Li, T.H. Luan, and X. Shen, "Channel Allocation for Smooth Video Delivery over Cognitive Radio Networks," in Proc. of IEEE GLOBECOM, Dec. 2010. 
[18] B. Guan and Y. He, "Optimal resource allocation for video streaming over cognitive radio networks," in Proc. of IEEE MMSP, Oct. 2011.

[19] F. Wang, M. Krunz, and S. Cui, "Spectrum sharing in cognitive radio networks," in Proc. IEEE Conf. Comput. Commun., pp.882-887, Jan. 2008.

[20] J. Mitola III and G. Maguire Jr, “Cognitive Radio: Making Software Radios More Personal,” Personal Communications, IEEE, vol. 6, no. 4, pp. 13-18, 1999.

[21] R.W. Thomas, L.A. DaSilva, A.B. MacKenzie, "Cognitive networks," in Proc. IEEE DySPAN 2005, pp. 352-360, Nov. 2005.

[22] T. V. Krishna and A. Das, "A survey on MAC protocols in OSA networks," Computer Networks Journal (Elsevier), vol. 53, no. 9, pp. 1377-1394, 2009.

[23] D. Cabric , and R. Brodersen, "Physical layer design issues unique to cognitive radio systems," in Proc. 2005 IEEE 16th Intl. Symp. PIMRC, 2005

[24] D. Cabric, I. O’Donnell, M.-W. Chen, and R. Brodersen, "Spectrum Sharing Radios," IEEE Circuits \& Sys. Mag., pp. 30-45, Second Quarter 2006

[25] R. H. Walden, "Analog-to-Digital Converters Survey and Analysis," IEEE J. Sel. Areas Comm., April 1999

[26] Y. Chen, Q. Zhao, and A. Swami, “Joint Design and Separation Principle for Opportunistic Spectrum Access," in Proc. of IEEE Asilomar Conference on Signals, Systems, and Computers, Oct. 2006. 
[27] M. Oner and F. Jondral, "On the extraction of the channel allocation information in spectrum pooling systems,” IEEE J. Sel. Areas Comm., vol. 25, no. 3, pp. 558-565, April 2007

[28] T. Banerjee, C. Ghosh, and D. P. Agrawal, "Wireless sensor based dynamic channel selection in cellular communication by cognitive radio approach," in Proc. 1st International Conference on Cognitive Radio Oriented Wireless Networks and Communications, June 2006

[29] C. T. Chou, S. Shankar. N, H. Kim, and K. G. Shin, "What and how much to gain by spectrum agile?," IEEE J. Sel. Areas Comm., vol. 25, no. 3, pp. 576-588, April 2007

[30] A. R. Fattahi, F. Fu, M. v. d. Schaar, and F. Paganini, "Mechanism based resource allocation for multimedia transmission over spectrum agile wireless networks," IEEE J. Sel. Areas Comm., vol. 25, no. 3, pp. 601-612, April 2007.

[31] T. Fujii and Y. Suzuki, “Ad-Hoc Cognitive Radio,” in Proc. of DySPAN, 2005.

[32] A. Sahai and N. Hoven, and R. Tandra, "Some fundamental limits on cognitive radio," in Proc. of Allerton Conference on Communication Control and Computing, Oct. 2004.

[33] D. Cabric, S. M. Mishra, and R. W. Brodersen, "Implementation issues in spectrum sensing for cognitive radios," in Proc. of the Asilomar Conference on Signals, Systems, and Computers, pp. 772 776, 2004.

[34] B. Wild and K. Ramchandran, "Detecting primary receivers for cognitive radio applications," in Proc. of DySPAN, Nov. 2005. 
[35] A. Ghasemi and E. Sousa, "Collaborative Spectrum Sensing for Opportunistic Access in Fading Environments," in Proc. of DySPAN, Nov. 2005

[36] H. Zheng and C. Peng, "Collaboration and Fairness in Opportunistic Spectrum Access," in Proc. of ICC, 2005.

[37] S. Geirhofer, L. Tong, and B. Sadler, "Dynamic spectrum access in WLAN channels: empirical model and its stochastic analysis," in Proc. of TAPAS, August 2006.

[38] A. Alshamrani, L. Xie, and X. Shen, "Adaptive admission control and channel allocation policy in cooperative ad hoc opportunistic spectrum networks," IEEE Transactions on Vehicular Technology, to appear.

[39] D. Hu, S. Mao, and J. H. Reed, "On video multicast in cognitive radio networks," in Proc. of IEEE INFOCOM, 2009.

[40] S. Boyd and L. Vandenberghe, Convex Optimization. Cambridge University Press, 2004.

[41] L. Le and E. Hossain, "Resource Allocation for Spectrum Underlay in Cognitive Radio Networks," IEEE Trans. Wireless Comm., vol. 7, no. 12, pp. 5306-5315, Dec. 2008.

[42] N.U. Prabhu, Foundation of Queuing Theory, Springer Press, 1997.

[43] K. B. S. Manosha and N.Rajatheva, "Joint power and rate control for spectrum underlay in cognitive radio networks with a novel pricing scheme," in Proc. IEEE Veh. Technol. Conf., pp.1-5, Sep. 2010. 
[44] R.D. Yates and D.J. Goodman, Probability and stochastic Processes, a friendly introduction for electrical and computer engineering, 2nd Edition, John Wiley \& Sons Inc., 2004.

[45] S. Boyd, S.J. Kim, L. Vandenberghe, and A. Hassibi, "A Tutorial on Geometric Programming," Optimization and Engineering, vol. 8, no. 1, pp. 67-127, Apr. 2007.

[46] S. Boyd and L. Vandenberghe, Convex Optimization, Cambridge University Press, 2004.

[47] J. Reichel, H. Schwarz, and M. Wien, Joint Scalable Video Model 11 (JSVM 11), Joint Video Team, Doc. JVT-X202, July 2007.

[48] Streaming media, Wikipedia, http://en.wikipedia.org/wiki/Streaming_media

[49] Ch. Z. Patrikakis, N. Papaoulakis, Ch. Stefanoudaki, M. S. Nunes, "Streaming content wars: Download and play strikes back," In Proc. of Personalization in Media Delivery Platforms Workshop, pp.218-226, 2009.

[50] C. Krasic, K. Li, and J. Walpole, "The case for streaming multimedia with TCP," Computer Science, Springer Press, pp. 213 -218, 2001. 


\section{List of Publications}

The publications based on the work of this thesis are listed below:

\section{Conference Papers}

- Bo Guan, Yifeng He, "Optimal resource allocation for video streaming over cognitive radio networks", In Proc. of IEEE MMSP, pp.160-165, Hangzhou, China, Dec. 2011

- Bo Guan, Yifeng He, "Optimal resource allocation for multi-layered video streaming over multi-channel cognitive radio networks", Submitted to IEEE IUCC, Liverpool, UK, June 2012 